\title{
Relative Density of the Random $r$-Factor Proximity Catch Digraph for Testing Spatial Patterns of Segregation and Association
}

\author{
Elvan Ceyhan, Carey E. Priebe, EG John C. Wierman \\ Johns Hopkins University, Baltimore
}

November 3, 2018

\begin{abstract}
Statistical pattern classification methods based on data-random graphs were introduced recently. In this approach, a random directed graph is constructed from the data using the relative positions of the data points from various classes. Different random graphs result from different definitions of the proximity region associated with each data point and different graph statistics can be employed for data reduction. The approach used in this article is based on a parameterized family of proximity maps determining an associated family of data-random digraphs. The relative arc density of the digraph is used as the summary statistic, providing an alternative to the domination number employed previously. An important advantage of the relative arc density is that, properly re-scaled, it is a $U$-statistic, facilitating analytic study of its asymptotic distribution using standard $U$-statistic central limit theory. The approach is illustrated with an application to the testing of spatial patterns of segregation and association. Knowledge of the asymptotic distribution allows evaluation of the Pitman and Hodges-Lehmann asymptotic efficacies, and selection of the proximity map parameter to optimize efficiency. Furthermore the approach presented here also has the advantage of validity for data in any dimension.
\end{abstract}

\section{Introduction}

Classification and clustering have received considerable attention in the statistical literature. In recent years, a new classification approach has been developed which is based on the relative positions of the data points from various classes. Priebe et al. introduced the class cover catch digraphs (CCCD) in $\mathbb{R}$ and gave the exact and the asymptotic distribution of the domination number of the CCCD Priebe et al. (2001). DeVinney et al. DeVinney et al. (2002), Marchette and Priebe Marchette and Priebe (2003), Priebe et al. Priebe et al. (2003b), Priebe et al. (2003a) applied the concept in higher dimensions and demonstrated relatively good performance of CCCD in classification. The methods employed involve data reduction (condensing) by using approximate minimum dominating sets as prototype sets (since finding the exact minimum dominating set is an NP-hard problem - in particular for CCCD). Furthermore the exact and the asymptotic distribution of the domination number of the CCCD are not analytically tractable in multiple dimensions.

Ceyhan and Priebe introduced the central similarity proximity map and $r$-factor proximity maps and the associated random digraphs in Cevhan and Priebe (2003a) and Cevhan and Priebe (2003b), respectively. In both cases, the space is partitioned by the Delaunay tessellation which is the Delaunay triangulation in $\mathbb{R}^{2}$. In each triangle, a family of data-random proximity catch digraphs is constructed based on the proximity of the points to each other. The advantages of the $r$-factor proximity catch digraphs are that an exact minimum dominating set can be found in polynomial time and the asymptotic distribution of the domination number is analytically tractable. The latter is then used to test segregation and association of points of different classes in Cevhan and Priebe (2003b). Segregation and assocation are two patterns that describe the spatial relation between two or more classes. See Section 2.5 for more detail. 
In this article, we employ a different statistic, namely the relative $(\operatorname{arc})$ density, that is the proportion of all possible arcs (directed edges) which are present in the data random digraph. This test statistic has the advantage that, properly rescaled, it is a $U$-statistic. Two plain classes of alternative hypotheses, for segregation and association, are defined in Section 2.5. The asymptotic distributions under both the null and the alternative hypotheses are determined in Section 3 by using standard $U$-statistic central limit theory. Pitman and Hodges-Lehman asymptotic efficacies are analyzed in Sections 4.3 and 4.4 respectively. This test is related to the available tests of segregation and association in the ecology literature, such as Pielou's test and Ripley's test. See discussion in Section [ for more detail. Our approach is valid for data in any dimension, but for simplicity of expression and visualization, will be described for two-dimensional data.

\section{Preliminaries}

\subsection{Proximity Maps}

Let $(\Omega, \mathcal{M})$ be a measurable space and consider a function $N: \Omega \times 2^{\Omega} \rightarrow 2^{\Omega}$, where $2^{\Omega}$ represents the power set of $\Omega$. Then given $\mathcal{Y} \subseteq \Omega$, the proximity map $N_{\mathcal{Y}}(\cdot)=N(\cdot, \mathcal{Y}): \Omega \rightarrow \wp(\Omega)$ associates with each point $x \in \Omega$ a proximity region $N_{\mathcal{Y}}(x) \subset \Omega$. Typically, $N$ is chosen to satisfy $x \in N_{\mathcal{Y}}(x)$ for all $x \in \Omega$. The use of the adjective proximity comes form thinking of the region $N_{\mathcal{Y}}(x)$ as representing a neighborhood of points "close" to $x$. (Jaromczyk and Toussaint (1992); Toussaint (1980).)

\section{$2.2 \quad r$-Factor Proximity Maps}

We now briefly define $r$-factor proximity maps. (See Ceyhan and Priebe Cevhan and Priebe (2003b) for more details). Let $\Omega=\mathbb{R}^{2}$ and let $\mathcal{Y}=\left\{\mathrm{y}_{1}, \mathrm{y}_{2}, \mathrm{y}_{3}\right\} \subset \mathbb{R}^{2}$ be three non-collinear points. Denote by $T(\mathcal{Y})$ the triangle - including the interior - formed by the three points (i.e. $T(\mathcal{Y})$ is the convex hull of $\mathcal{Y}$ ). For $r \in[1, \infty]$, define $N_{\mathcal{Y}}^{r}$ to be the $r$-factor proximity map as follows; see also Figure 1 Using line segments from the center of mass (centroid) of $T(\mathcal{Y})$ to the midpoints of its edges, we partition $T(\mathcal{Y})$ into "vertex regions" $R\left(\mathrm{y}_{1}\right), R\left(\mathrm{y}_{2}\right)$, and $R\left(\mathrm{y}_{3}\right)$. For $x \in T(\mathcal{Y}) \backslash \mathcal{Y}$, let $v(x) \in \mathcal{Y}$ be the vertex in whose region $x$ falls, so $x \in R(v(x))$. If $x$ falls on the boundary of two vertex regions, we assign $v(x)$ arbitrarily to one of the adjacent regions. Let $e(x)$ be the edge of $T(\mathcal{Y})$ opposite $v(x)$. Let $\ell(x)$ be the line parallel to $e(x)$ through $x$. Let $d(v(x), \ell(x))$ be the Euclidean (perpendicular) distance from $v(x)$ to $\ell(x)$. For $r \in[1, \infty)$, let $\ell_{r}(x)$ be the line parallel to $e(x)$ such that $d\left(v(x), \ell_{r}(x)\right)=r d(v(x), \ell(x))$ and $d\left(\ell(x), \ell_{r}(x)\right)<d\left(v(x), \ell_{r}(x)\right)$. Let $T_{r}(x)$ be the triangle similar to and with the same orientation as $T(\mathcal{Y})$ having $v(x)$ as a vertex and $\ell_{r}(x)$ as the opposite edge. Then the $r$-factor proximity region $N_{\mathcal{Y}}^{r}(x)$ is defined to be $T_{r}(x) \cap T(\mathcal{Y})$. Notice that $r \geq 1$ implies $x \in N_{\mathcal{Y}}^{r}(x)$. Note also that $\lim _{r \rightarrow \infty} N_{\mathcal{Y}}^{r}(x)=T(\mathcal{Y})$ for all $x \in T(\mathcal{Y}) \backslash \mathcal{Y}$, so we define $N_{\mathcal{Y}}^{\infty}(x)=T(\mathcal{Y})$ for all such $x$. For $x \in \mathcal{Y}$, we define $N_{\mathcal{Y}}^{r}(x)=\{x\}$ for all $r \in[1, \infty]$.

\subsection{Data-Random Proximity Catch Digraphs}

If $\mathcal{X}_{n}:=\left\{X_{1}, X_{2}, \cdots, X_{n}\right\}$ is a set of $\Omega$-valued random variables, then the $N_{\mathcal{Y}}\left(X_{i}\right), i=1, \cdots, n$, are random sets. If the $X_{i}$ are independent and identically distributed, then so are the random sets $N_{\mathcal{Y}}\left(X_{i}\right)$.

In the case of an $r$-factor proximity map, notice that if $X_{i} \stackrel{i i d}{\sim} F$ and $F$ has a non-degenerate twodimensional probability density function $f$ with support $(f) \subseteq T(\mathcal{Y})$, then the special case in the construction of $N_{\mathcal{Y}}^{r}-X$ falls on the boundary of two vertex regions - occurs with probability zero.

The proximities of the data points to each other are used to construct a digraph. A digraph is a directed graph; i.e. a graph with directed edges from one vertex to another based on a binary relation. Define the data-random proximity catch digraph $D$ with vertex set $\mathcal{V}=\left\{X_{1}, \cdots, X_{n}\right\}$ and arc set $\mathcal{A}$ by $\left(X_{i}, X_{j}\right) \in \mathcal{A} \Longleftrightarrow X_{j} \in N_{\mathcal{Y}}\left(X_{i}\right)$. Since this relationship is not symmetric, a digraph is needed rather than 


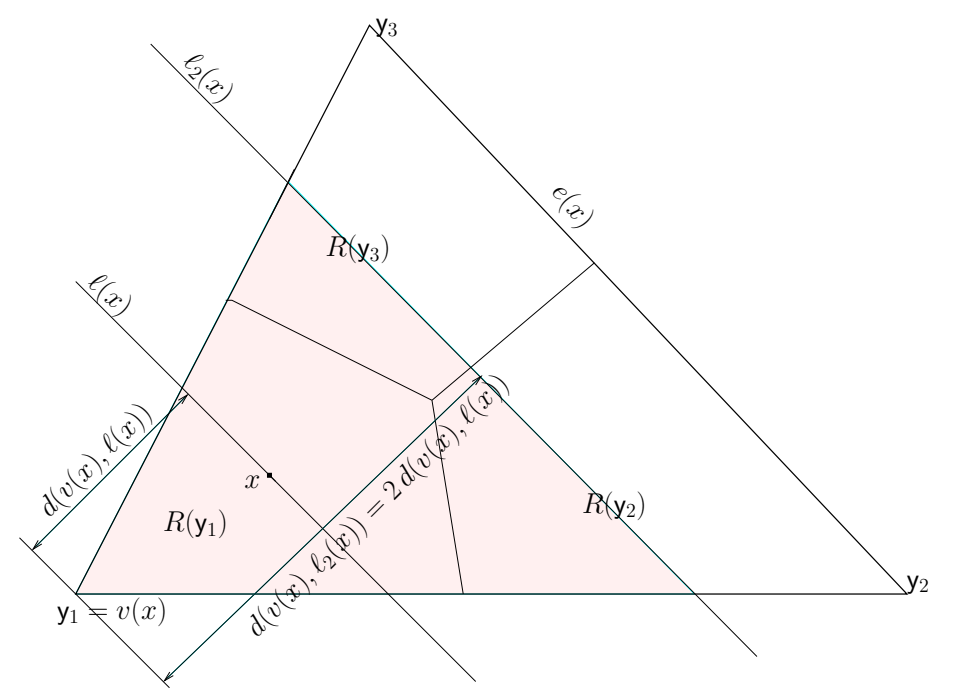

Figure 1: Construction of $r$-factor proximity region, $N_{\mathcal{Y}}^{2}(x)$ (shaded region).

a graph. The random digraph $D$ depends on the (joint) distribution of the $X_{i}$ and on the map $N_{\mathcal{Y}}$.

\subsection{Relative Density}

The relative arc density of a digraph $D=(\mathcal{V}, \mathcal{A})$ of order $|\mathcal{V}|=n$, denoted $\rho(D)$, is defined as

$$
\rho(D)=\frac{|\mathcal{A}|}{n(n-1)}
$$

where $|\cdot|$ denotes the set cardinality functional Janson et al. (2000).

Thus $\rho(D)$ represents the ratio of the number of arcs in the digraph $D$ to the number of arcs in the complete symmetric digraph of order $n$, which is $n(n-1)$. For brevity of notation we use relative density rather than relative arc density henceforth.

If $X_{1}, \cdots, X_{n} \stackrel{\text { iid }}{\sim} F$ the relative density of the associated data-random proximity catch digraph $D$, denoted $\rho\left(\mathcal{X}_{n} ; h, N_{\mathcal{Y}}\right)$, is a $U$-statistic,

$$
\rho\left(\mathcal{X}_{n} ; h, N_{\mathcal{Y}}\right)=\frac{1}{n(n-1)} \sum_{i<j} \sum_{h}\left(X_{i}, X_{j} ; N_{\mathcal{Y}}\right)
$$

where

$$
\begin{aligned}
h\left(X_{i}, X_{j} ; N_{\mathcal{Y}}\right) & =\mathbf{I}\left\{\left(X_{i}, X_{j}\right) \in \mathcal{A}\right\}+\mathbf{I}\left\{\left(X_{j}, X_{i}\right) \in \mathcal{A}\right\} \\
& =\mathbf{I}\left\{X_{j} \in N_{\mathcal{Y}}\left(X_{i}\right)\right\}+\mathbf{I}\left\{X_{i} \in N_{\mathcal{Y}}\left(X_{j}\right)\right\},
\end{aligned}
$$

where $\mathbf{I}(\cdot)$ is the indicator function. We denote $h\left(X_{i}, X_{j} ; N_{\mathcal{Y}}\right)$ as $h_{i j}$ for brevity of notation. Although the digraph is asymmetric, $h_{i j}$ is defined as the number of arcs in $D$ between vertices $X_{i}$ and $X_{j}$, in order to produce a symmetric kernel with finite variance Lehmann (1988).

The random variable $\rho_{n}:=\rho\left(\mathcal{X}_{n} ; h, N_{\mathcal{Y}}\right)$ depends on $n$ and $N_{\mathcal{Y}}$ explicitly and on $F$ implicitly. The expectation $\mathbf{E}\left[\rho_{n}\right]$, however, is independent of $n$ and depends on only $F$ and $N_{\mathcal{Y}}$ : 
The variance $\operatorname{Var}\left[\rho_{n}\right]$ simplifies to

$$
0 \leq \operatorname{Var}\left[\rho_{n}\right]=\frac{1}{2 n(n-1)} \operatorname{Var}\left[h_{12}\right]+\frac{n-2}{n(n-1)} \operatorname{Cov}\left[h_{12}, h_{13}\right] \leq 1 / 4 .
$$

A central limit theorem for $U$-statistics Lehmann (1988) yields

$$
\sqrt{n}\left(\rho_{n}-\mathbf{E}\left[\rho_{n}\right]\right) \stackrel{\mathcal{L}}{\longrightarrow} \mathcal{N}\left(0, \mathbf{C o v}\left[h_{12}, h_{13}\right]\right)
$$

provided $\operatorname{Cov}\left[h_{12}, h_{13}\right]>0$. The asymptotic variance of $\rho_{n}$, $\operatorname{Cov}\left[h_{12}, h_{13}\right]$, depends on only $F$ and $N_{\mathcal{Y}}$. Thus, we need determine only $\mathbf{E}\left[h_{12}\right]$ and $\mathbf{C o v}\left[h_{12}, h_{13}\right]$ in order to obtain the normal approximation

$$
\rho_{n} \stackrel{\operatorname{approx}}{\sim} \mathcal{N}\left(\mathbf{E}\left[\rho_{n}\right], \operatorname{Var}\left[\rho_{n}\right]\right)=\mathcal{N}\left(\frac{\mathbf{E}\left[h_{12}\right]}{2}, \frac{\mathbf{C o v}\left[h_{12}, h_{13}\right]}{n}\right) \text { for large } n .
$$

\subsection{Null and Alternative Hypotheses}

In a two class setting, the phenomenon known as segregation occurs when members of one class have a tendency to repel members of the other class. For instance, it may be the case that one type of plant does not grow well in the vicinity of another type of plant, and vice versa. This implies, in our notation, that $X_{i}$ are unlikely to be located near any elements of $\mathcal{Y}$. Alternatively, association occurs when members of one class have a tendency to attract members of the other class, as in symbiotic species, so that the $X_{i}$ will tend to cluster around the elements of $\mathcal{Y}$, for example. See, for instance, Dixon (1994), Coomes et al. (1999). The null hypothesis for spatial patterns have been a contraversial topic in ecology from the early days. Gotelli and Graves Gotelli and Graves (1996) have collected a voluminous literature to present a comprehensive analysis of the use and misuse of null models in ecology community. They also define and attempt to clarify the null model concept as "a pattern-generating model that is based on randomization of ecological data or random sampling from a known or imagined distribution. . . . The randomization is designed to produce a pattern that would be expected in the absence of a particular ecological mechanism." In other words, the hypothesized null models can be viewed as "thought experiments," which is conventially used in the physical sciences, and these models provide a statistical baseline for the analysis of the patterns. For statistical testing for segregation and association, the null hypothesis we consider is a type of complete spatial randomness; that is,

$$
H_{0}: X_{i} \stackrel{i i d}{\sim} \mathcal{U}(T(\mathcal{Y}))
$$

where $\mathcal{U}(T(\mathcal{Y}))$ is the uniform distribution on $T(\mathcal{Y})$. If it is desired to have the sample size be a random variable, we may consider a spatial Poisson point process on $T(\mathcal{Y})$ as our null hypothesis.

We define two classes of alternatives, $H_{\epsilon}^{S}$ and $H_{\epsilon}^{A}$ with $\epsilon \in(0, \sqrt{3} / 3)$, for segregation and association, respectively. For $\mathrm{y} \in \mathcal{Y}$, let $e(\mathrm{y})$ denote the edge of $T(\mathcal{Y})$ opposite vertex $\mathrm{y}$, and for $x \in T(\mathcal{Y})$ let $\ell_{\mathrm{y}}(x)$ denote the line parallel to $e(\mathrm{y})$ through $x$. Then define $T(\mathrm{y}, \epsilon)=\left\{x \in T(\mathcal{Y}): d\left(\mathrm{y}, \ell_{\mathrm{y}}(x)\right) \leq \epsilon\right\}$. Let $H_{\epsilon}^{S}$ be the model under which $X_{i} \stackrel{i i d}{\sim} \mathcal{U}\left(T(\mathcal{Y}) \backslash \cup_{\mathrm{y} \in \mathcal{Y}} T(\mathrm{y}, \epsilon)\right)$ and $H_{\epsilon}^{A}$ be the model under which $X_{i} \stackrel{i i d}{\sim} \mathcal{U}\left(\cup_{\mathrm{y} \in \mathcal{Y}} T(\mathrm{y}, \sqrt{3} / 3-\epsilon)\right)$. Thus the segregation model excludes the possibility of any $X_{i}$ occurring near a $\mathrm{y}_{j}$, and the association model requires that all $X_{i}$ occur near a $\mathrm{y}_{j}$. The $\sqrt{3} / 3-\epsilon$ in the definition of the association alternative is so that $\epsilon=0$ yields $H_{0}$ under both classes of alternatives.

Remark: These definitions of the alternatives are given for the standard equilateral triangle. The geometry invariance result of Theorem 1 from Section 3 still holds under the alternatives, in the following sense. If, in an arbitrary triangle, a small percentage $\delta \cdot 100 \%$ where $\delta \in(0,4 / 9)$ of the area is carved away as forbidden from each vertex using line segments parallel to the opposite edge, then under the transformation to the standard equilateral triangle this will result in the alternative $H_{\sqrt[3]{3 \delta / 4}}^{S}$. This argument is for segregation with $\delta<1 / 4$; a similar construction is available for the other cases. 


\section{Asymptotic Normality Under the Null and Alternative Hy- potheses}

First we present a "geometry invariance" result which allows us to assume $T(\mathcal{Y})$ is the standard equilateral triangle, $T((0,0),(1,0),(1 / 2, \sqrt{3} / 2))$, thereby simplifying our subsequent analysis.

Theorem 1: Let $\mathcal{Y}=\left\{\mathrm{y}_{1}, \mathrm{y}_{2}, \mathrm{y}_{3}\right\} \subset \mathbb{R}^{2}$ be three non-collinear points. For $i=1, \cdots, n$ let $X_{i} \stackrel{i i d}{\sim}$ $F=\mathcal{U}(T(\mathcal{Y}))$, the uniform distribution on the triangle $T(\mathcal{Y})$. Then for any $r \in[1, \infty]$ the distribution of $\rho\left(\mathcal{X}_{n} ; h, N_{\mathcal{Y}}^{r}\right)$ is independent of $\mathcal{Y}$, hence the geometry of $T(\mathcal{Y})$.

Proof: A composition of translation, rotation, reflections, and scaling will transform any given triangle $T_{o}=T\left(\mathrm{y}_{1}, \mathrm{y}_{2}, \mathrm{y}_{3}\right)$ into the "basic" triangle $T_{b}=T\left((0,0),(1,0),\left(c_{1}, c_{2}\right)\right)$ with $0<c_{1} \leq 1 / 2, c_{2}>0$ and $\left(1-c_{1}\right)^{2}+c_{2}^{2} \leq 1$, preserving uniformity. The transformation $\phi_{e}: \mathbb{R}^{2} \rightarrow \mathbb{R}^{2}$ given by $\phi_{e}(u, v)=$ $\left(u+\frac{1-2 c_{1}}{\sqrt{3}} v, \frac{\sqrt{3}}{2 c_{2}} v\right)$ takes $T_{b}$ to the equilateral triangle $T_{e}=T((0,0),(1,0),(1 / 2, \sqrt{3} / 2))$. Investigation of the Jacobian shows that $\phi_{e}$ also preserves uniformity. Furthermore, the composition of $\phi_{e}$ with the rigid motion transformations maps the boundary of the original triangle $T_{o}$ to the boundary of the equilateral triangle $T_{e}$, the median lines of $T_{o}$ to the median lines of $T_{e}$, and lines parallel to the edges of $T_{o}$ to lines parallel to the edges of $T_{e}$. Since the joint distribution of any collection of the $h_{i j}$ involves only probability content of unions and intersections of regions bounded by precisely such lines, and the probability content of such regions is preserved since uniformity is preserved, the desired result follows.

Based on Theorem 1 and our uniform null hypothesis, we may assume that $T(\mathcal{Y})$ is the standard equilateral triangle with $\mathcal{Y}=\{(0,0),(1,0),(1 / 2, \sqrt{3} / 2)\}$ henceforth.

For our $r$-factor proximity map and uniform null hypothesis, the asymptotic null distribution of $\rho_{n}(r)=$ $\rho\left(\mathcal{X}_{n} ; h, N_{\mathcal{Y}}^{r}\right)$ can be derived as a function of $r$. Let $\mu(r):=\mathbf{E}\left[\rho_{n}(r)\right]$ and $\nu(r):=\mathbf{C o v}\left[h_{12}, h_{13}\right]$. Notice that $\mu(r)=\mathbf{E}\left[h_{12}\right] / 2=P\left(X_{2} \in N_{\mathcal{Y}}^{r}\left(X_{1}\right)\right)$ is the probability of an arc occurring between any pair of vertices.

\subsection{Asymptotic Normality under the Null Hypothesis}

By detailed geometric probability calculations, provided in Appendix 1, the mean and the asymptotic variance of the relative density of the $r$-factor proximity catch digraph can explicitly be computed. The central limit theorem for $U$-statistics then establishes the asymptotic normality under the uniform null hypothesis. These results are summarized in the following theorem.

Theorem 2: For $r \in[1, \infty)$,

$$
\frac{\sqrt{n}\left(\rho_{n}(r)-\mu(r)\right)}{\sqrt{\nu(r)}} \stackrel{\mathcal{L}}{\longrightarrow} \mathcal{N}(0,1)
$$

where

$$
\mu(r)=\left\{\begin{array}{lll}
\frac{37}{216} r^{2} & \text { for } & r \in[1,3 / 2) \\
-\frac{1}{8} r^{2}+4-8 r^{-1}+\frac{9}{2} r^{-2} & \text { for } & r \in[3 / 2,2) \\
1-\frac{3}{2} r^{-2} & \text { for } & r \in[2, \infty)
\end{array}\right.
$$

and

$$
\nu(r)=\nu_{1}(r) \mathbf{I}(r \in[1,4 / 3))+\nu_{2}(r) \mathbf{I}(r \in[4 / 3,3 / 2))+\nu_{3}(r) \mathbf{I}(r \in[3 / 2,2))+\nu_{4}(r) \mathbf{I}(r \in[2, \infty])
$$


with

$$
\begin{aligned}
\nu_{1}(r) & =\frac{3007 r^{10}-13824 r^{9}+898 r^{8}+77760 r^{7}-117953 r^{6}+48888 r^{5}-24246 r^{4}+60480 r^{3}-38880 r^{2}+3888}{58320 r^{4}}, \\
\nu_{2}(r) & =\frac{5467 r^{10}-37800 r^{9}+61912 r^{8}+46588 r^{6}-191520 r^{5}+13608 r^{4}+241920 r^{3}-155520 r^{2}+15552}{233280 r^{4}}, \\
\nu_{3}(r) & =-\left[7 r^{12}-72 r^{11}+312 r^{10}-5332 r^{8}+15072 r^{7}+13704 r^{6}-139264 r^{5}+273600 r^{4}-242176 r^{3}\right. \\
& \left.+103232 r^{2}-27648 r+8640\right] /\left[960 r^{6}\right], \\
\nu_{4}(r) & =\frac{15 r^{4}-11 r^{2}-48 r+25}{15 r^{6}} .
\end{aligned}
$$

For $r=\infty, \rho_{n}(r)$ is degenerate.

See Appendix 1 for the proof.

Consider the form of the mean and variance functions, which are depicted in Figure 2. Note that $\mu(r)$ is monotonically increasing in $r$, since the proximity region of any data point increases with $r$. In addition, $\mu(r) \rightarrow 1$ as $r \rightarrow \infty$, since the digraph becomes complete asymptotically, which explains why $\rho_{n}(r)$ is degenerate, i.e. $\nu(r)=0$, when $r=\infty$. Note also that $\mu(r)$ is continuous, with the value at $r=1$ $\mu(1)=37 / 216$.

Regarding the asymptotic variance, note that $\nu(r)$ is continuous in $r$ with $\lim _{r \rightarrow \infty} \nu(r)=0$ and $\nu(1)=$ $34 / 58320 \approx .000583$ and observe that $\sup _{r \geq 1} \nu(r) \approx .1305$ at $\operatorname{argsup}_{r \geq 1} \nu(r) \approx 2.045$.
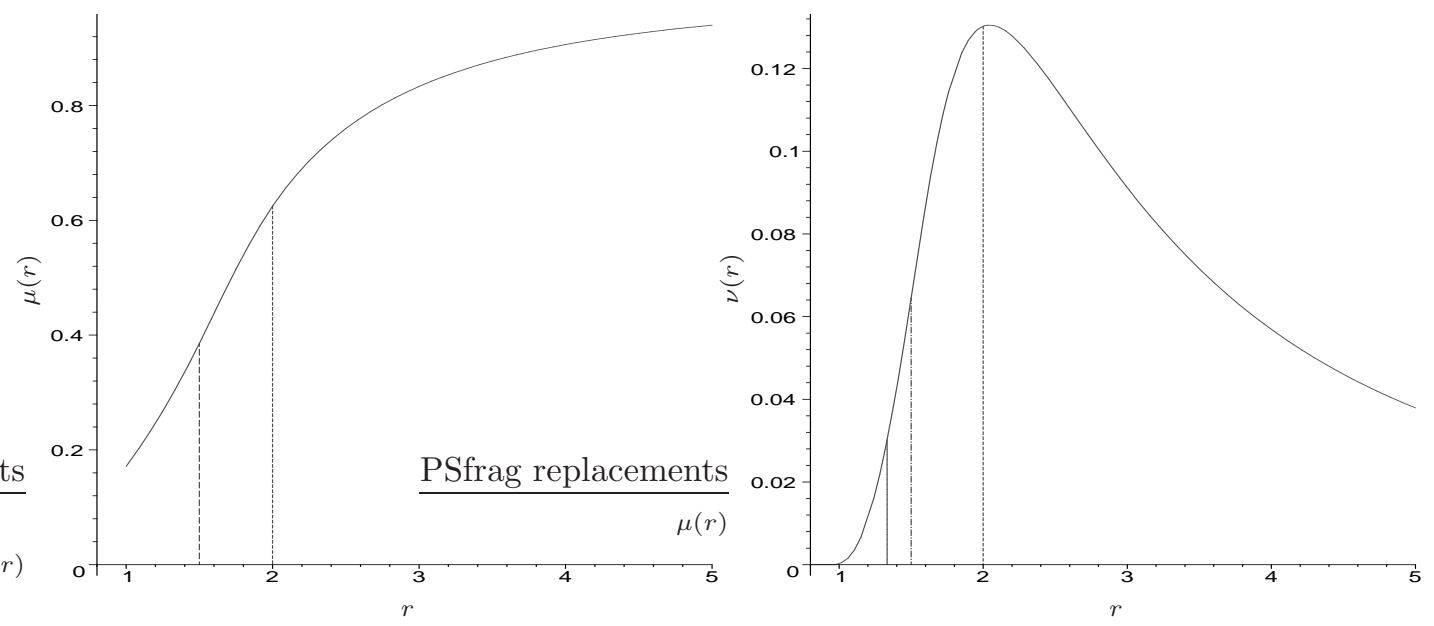

Figure 2: Asymptotic null mean $\mu(r)$ (left) and variance $\nu(r)$ (right), from Equations (8) and (9) in Theorem 2 , respectively. The vertical lines indicate the endpoints of the intervals in the piecewise definition of the functions. Notice that the vertical axes are differently scaled.

To illustrate the limiting distribution, $r=2$ yields

$$
\frac{\sqrt{n}\left(\rho_{n}(2)-\mu(2)\right)}{\sqrt{\nu(2)}}=\sqrt{\frac{192 n}{25}}\left(\rho_{n}(2)-\frac{5}{8}\right) \stackrel{\mathcal{L}}{\longrightarrow} \mathcal{N}(0,1)
$$

or equivalently

$$
\rho_{n}(2) \stackrel{\text { approx }}{\sim} \mathcal{N}\left(\frac{5}{8}, \frac{25}{192 n}\right)
$$

Figure 3 indicates that, for $r=2$, the normal approximation is accurate even for small $n$ (although kurtosis may be indicated for $n=10$ ). Figure 4 demonstrates, however, that severe skewness obtains for small values of $n$, and extreme values of $r$. The finite sample variance in Equation 4 and skewness may be derived analytically in much the same way as was Cov $\left[h_{12}, h_{13}\right]$ for the asymptotic variance. In fact, the 
exact distribution of $\rho_{n}(r)$ is, in principle, available by successively conditioning on the values of the $X_{i}$. Alas, while the joint distribution of $h_{12}, h_{13}$ is available, the joint distribution of $\left\{h_{i j}\right\}_{1 \leq i<j \leq n}$, and hence the calculation for the exact distribution of $\rho_{n}(r)$, is extraordinarily tedious and lengthy for even small values of $n$.
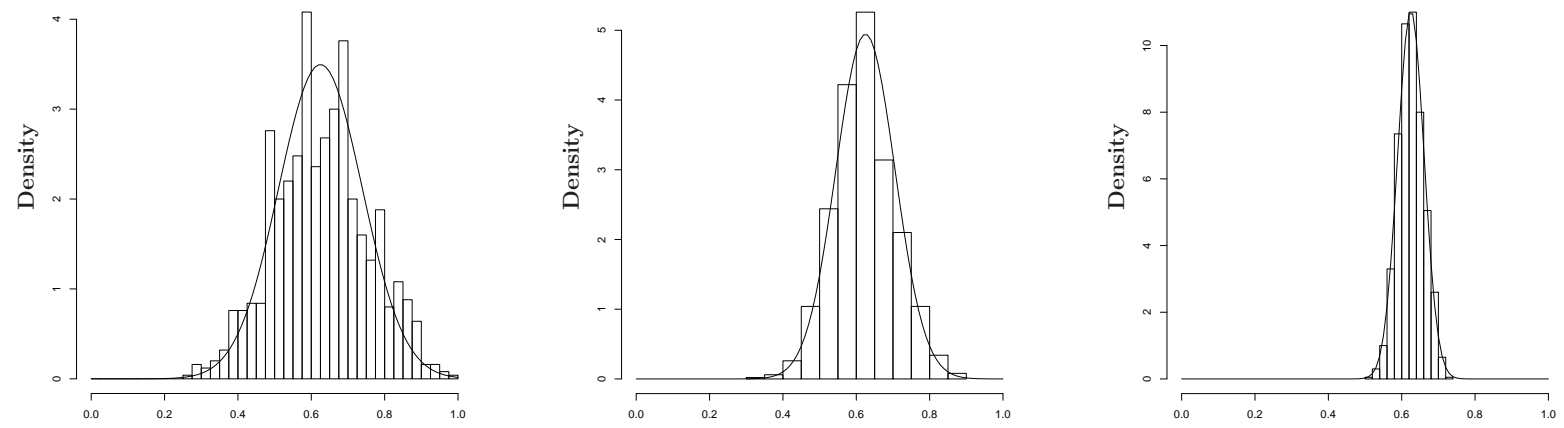

Figure 3: Depicted are the distributions of $\rho_{n}(2) \stackrel{\text { approx }}{\sim} \mathcal{N}\left(\frac{5}{8}, \frac{25}{192 n}\right)$ for $n=10,20,100$ (left to right). Histograms are based on 1000 Monte Carlo replicates. Solid curves represent the approximating normal densities given by Theorem 2. Again, note that the vertical axes are differently scaled.
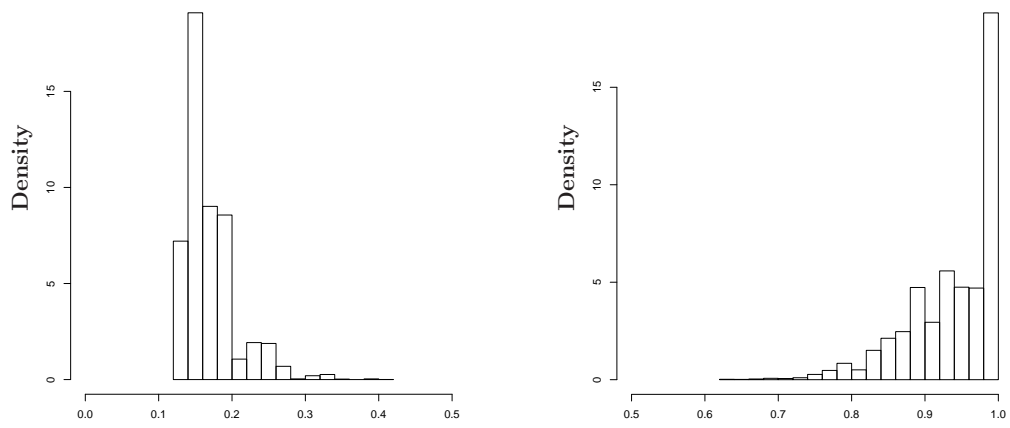

Figure 4: Depicted are the histograms for 10,000 Monte Carlo replicates of $\rho_{10}(1)$ (left) and $\rho_{10}(5)$ (right) indicating severe small sample skewness for extreme values of $r$.

Letting $H_{n}(r)=\sum_{i=1}^{n} h\left(X_{i}, X_{n+1}\right)$, the exact distribution of $\rho_{n}(r)$ can be evaluated based on the recurrence

$$
(n+1) n \rho_{n+1}(r) \stackrel{d}{=} n(n-1) \rho_{n}(r)+H_{n}(r)
$$

by noting that the conditional random variable $H_{n}(r) \mid X_{n+1}$ is the sum of $n$ independent and identically distributed random variables. Alas, this calculation is also tedious for large $n$.

\section{$3.2 \quad$ Asymptotic Normality Under the Alternatives}

Asymptotic normality of relative density of the proximity catch digraphs under the alternative hypotheses of segregation and association can be established by the same method as under the null hypothesis. Let $\mathbf{E}_{\epsilon}^{S}[\cdot]$ $\left(\mathbf{E}_{\epsilon}^{A}[\cdot]\right)$ be the expectation with respect to the uniform distribution under the segregation ( association ) alternatives with $\epsilon \in(0, \sqrt{3} / 3)$. 
Theorem 3: Let $\mu_{S}(r, \epsilon)$ (and $\mu_{A}(r, \epsilon)$ ) be the mean and $\nu_{S}(r, \epsilon)$ (and $\nu_{A}(r, \epsilon)$ ) be the covariance, $\operatorname{Cov}\left[h_{12}, h_{13}\right]$ for $r \in(0,1]$ and $\epsilon \in(0, \sqrt{3} / 3)$ under segregation (and association). Then under $H_{\epsilon}^{S}$, $\sqrt{n}\left(\rho_{n}(r)-\mu_{S}(r, \epsilon)\right) \stackrel{\mathcal{L}}{\longrightarrow} \mathcal{N}\left(0, \nu_{S}(r, \epsilon)\right)$ for the values of the pair $(r, \epsilon)$ for which $\nu_{S}(r, \epsilon)>0$. Likewise, under $H_{\epsilon}^{A}, \sqrt{n}\left(\rho_{n}(r)-\mu_{A}(r, \epsilon)\right) \stackrel{\mathcal{L}}{\longrightarrow} \mathcal{N}\left(0, \nu_{A}(r, \epsilon)\right)$ for the values of the pair $(r, \epsilon)$ for which $\nu_{A}(r, \epsilon)>0$.

Sketch of Proof: Under the alternatives, i.e. $\epsilon>0, \rho_{n}(r)$ is a $U$-statistic with the same symmetric kernel $h_{i j}$ as in the null case. The mean $\mu_{S}(r, \epsilon)=\mathbf{E}_{\epsilon}\left[\rho_{n}(r)\right]=\mathbf{E}_{\epsilon}\left[h_{12}\right] / 2$ (and $\mu_{A}(r, \epsilon)$ ), now a function of both $r$ and $\epsilon$, is again in [0,1]. The asymptotic variance $\nu_{S}(r, \epsilon)=\mathbf{C o v}_{\epsilon}\left[h_{12}, h_{13}\right]\left(\right.$ and $\left.\nu_{A}(r, \epsilon)\right)$, also a function of both $r$ and $\epsilon$, is bounded above by $1 / 4$, as before. The explicit forms of $\mu_{S}(r, \epsilon)$ and $\mu_{A}(r, \epsilon)$ is given, defined piecewise, in Appendix 2. Sample values of $\mu_{S}(r, \epsilon), \nu_{S}(r, \epsilon)$ and $\mu_{A}(r, \epsilon), \nu_{A}(r, \epsilon)$ are given in Appendix 3 for segregation with $\epsilon=\sqrt{3} / 4$ and for association with $\epsilon=\sqrt{3} / 12$. Thus asymptotic normality obtains provided $\nu_{S}(r, \epsilon)>0\left(\nu_{A}(r, \epsilon)>0\right)$; otherwise $\rho_{n}(r)$ is degenerate. Note that under $H_{\epsilon}^{S}$,

$$
\nu_{S}(r, \epsilon)>0 \text { for }(r, \epsilon) \in[1, \sqrt{3} /(2 \epsilon)) \times(0, \sqrt{3} / 4] \cup[1, \sqrt{3} / \epsilon-2) \times(\sqrt{3} / 4, \sqrt{3} / 3),
$$

and under $H_{\epsilon}^{A}$,

$$
\nu_{A}(r, \epsilon)>0 \text { for }(r, \epsilon) \in(1, \infty) \times(0, \sqrt{3} / 3) \cup\{1\} \times(0, \sqrt{3} / 12) .
$$

Notice that for the association class of alternatives any $r \in(1, \infty)$ yields asymptotic normality for all $\epsilon \in(0, \sqrt{3} / 3)$, while for the segregation class of alternatives only $r=1$ yields this universal asymptotic normality.

\section{The Test and Analysis}

The relative density of the proximity catch digraph is a test statistic for the segregation/association alternative; rejecting for extreme values of $\rho_{n}(r)$ is appropriate since under segregation we expect $\rho_{n}(r)$ to be large, while under association we expect $\rho_{n}(r)$ to be small. Using the test statistic

$$
R=\frac{\sqrt{n}\left(\rho_{n}(r)-\mu(r)\right)}{\sqrt{\nu(r)}}
$$

the asymptotic critical value for the one-sided level $\alpha$ test against segregation is given by

$$
z_{\alpha}=\Phi^{-1}(1-\alpha)
$$

where $\Phi(\cdot)$ is the standard normal distribution function. Against segregation, the test rejects for $R>z_{1-\alpha}$ and against association, the test rejects for $R<z_{\alpha}$.

\subsection{Consistency}

Theorem 4: The test against $H_{\epsilon}^{S}$ which rejects for $R>z_{1-\alpha}$ and the test against $H_{\epsilon}^{A}$ which rejects for $R<z_{\alpha}$ are consistent for $r \in[1, \infty)$ and $\epsilon \in(0, \sqrt{3} / 3)$.

Proof: Since the variance of the asymptotically normal test statistic, under both the null and the alternatives, converges to 0 as $n \rightarrow \infty$ (or is degenerate), it remains to show that the mean under the null, $\mu(r)=\mathbf{E}\left[\rho_{n}(r)\right]$, is less than (greater than) the mean under the alternative, $\mu_{S}(r, \epsilon)=E_{\epsilon}\left[\rho_{n}(r)\right]\left(\mu_{A}(r, \epsilon)\right)$ against segregation (association) for $\epsilon>0$. Whence it will follow that power converges to 1 as $n \rightarrow \infty$.

Detailed analysis of $\mu_{S}(r, \epsilon)$ and $\mu_{A}(r, \epsilon)$ in Appendix 2 indicates that under segregation $\mu_{S}(r, \epsilon)>\mu(r)$ for all $\epsilon>0$ and $r \in[1, \infty)$. Likewise, detailed analysis of $\mu_{A}(r, \epsilon)$ in Appendix 3 indicates that under association $\mu_{A}(r, \epsilon)<\mu(r)$ for all $\epsilon>0$ and $r \in[1, \infty)$. Hence the desired result follows for both alternatives.

In fact, the analysis of $\mu(r, \epsilon)$ under the alternatives reveals more than what is required for consistency. Under segregation, the analysis indicates that $\mu_{S}\left(r, \epsilon_{1}\right)<\mu_{S}\left(r, \epsilon_{2}\right)$ for $\epsilon_{1}<\epsilon_{2}$. Likewise, under association, the analysis indicates that $\mu_{A}\left(r, \epsilon_{1}\right)>\mu_{A}\left(r, \epsilon_{2}\right)$ for $\epsilon_{1}<\epsilon_{2}$. 


\subsection{Monte Carlo Power Analysis}
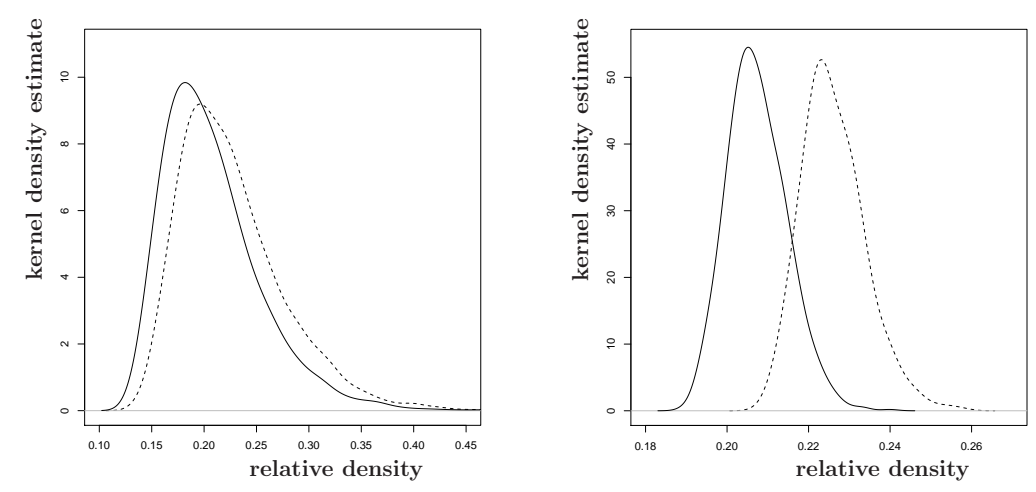

Figure 5: Two Monte Carlo experiments against the segregation alternative $H_{\sqrt{3} / 8}^{S}$. Depicted are kernel density estimates for $\rho_{n}(11 / 10)$ for $n=10$ (left) and $n=100$ (right) under the null (solid) and alternative (dashed).

In Figure 5] we present a Monte Carlo investigation against the segregation alternative $H_{\sqrt{3} / 8}^{S}$ for $r=$ $11 / 10$ and $n=10,100$. With $n=10$, the null and alternative probability density functions for $\rho_{10}(1.1)$ are very similar, implying small power (10,000 Monte Carlo replicates yield $\widehat{\beta}_{m c}^{S}=0.0787$, which is based on the empirical critical value). With $n=100$, there is more separation between null and alternative probability density functions; for this case, 1000 Monte Carlo replicates yield $\widehat{\beta}_{m c}^{S}=0.77$. Notice also that the probability density functions are more skewed for $n=10$, while approximate normality holds for $n=100$.

For a given alternative and sample size, we may consider analyzing the power of the test — using the asymptotic critical value - as a function of the proximity factor $r$. In Figure 6] we present a Monte Carlo investigation of power against $H_{\sqrt{3} / 8}^{S}$ and $H_{\sqrt{3} / 4}^{S}$ as a function of $r$ for $n=10$. The empirical significance level is about .05 for $r=2,3$ which have the empirical power $\widehat{\beta}_{10}^{S}(r, \sqrt{3} / 8) \approx .35$, and $\widehat{\beta}_{10}^{S}(r, \sqrt{3} / 4)=1$. So, for small sample sizes, moderate values of $r$ are more appropriate for normal approximation, as they yield the desired significance level and the more severe the segregation, the higher the power estimate.

In Figure 7. we present a Monte Carlo investigation against the association alternative $H_{\sqrt{3} / 12}^{A}$ for $r=$ $11 / 10$ and $n=10$ and 100. The analysis is same as in the analysis of the Figure 5 . In Figure 8, we present a Monte Carlo investigation of power against $H_{\sqrt{3} / 12}^{A}$ and $H_{5 \sqrt{3} / 24}^{A}$ as a function of $r$ for $n=10$. The empirical significance level is about .05 for $r=3 / 2,2,3,5$ which have the empirical power $\widehat{\beta}_{10}^{A}(r, \sqrt{3} / 12) \leq .35$ with maximum power at $r=2$, and $\widehat{\beta}_{10}^{A}(r, 5 \sqrt{3} / 24)=1$ at $r=3$. So, for small sample sizes, moderate values of $r$ are more appropriate for normal approximation, as they yield the desired significance level, and the more severe the association, the higher the power estimate.

\subsection{Pitman Asymptotic Efficacy}

Pitman asymptotic efficiency (PAE) provides for an investigation of "local asymptotic power" — local around $H_{0}$. This involves the limit as $n \rightarrow \infty$ as well as the limit as $\epsilon \rightarrow 0$. A detailed discussion of PAE can be found in Kendall and Stuart (1979) and Eeden (1963). For segregation or association alternatives the PAE is given by $\operatorname{PAE}\left(\rho_{n}(r)\right)=\frac{\left(\mu^{(k)}(r, \epsilon=0)\right)^{2}}{\nu(r)}$ where $k$ is the minimum order of the derivative with respect to $\epsilon$ for which $\mu^{(k)}(r, \epsilon=0) \neq 0$. That is, $\mu^{(k)}(r, \epsilon=0) \neq 0$ but $\mu^{(l)}(r, \epsilon=0)=0$ for $l=1,2, \ldots, k-1$. Then under 

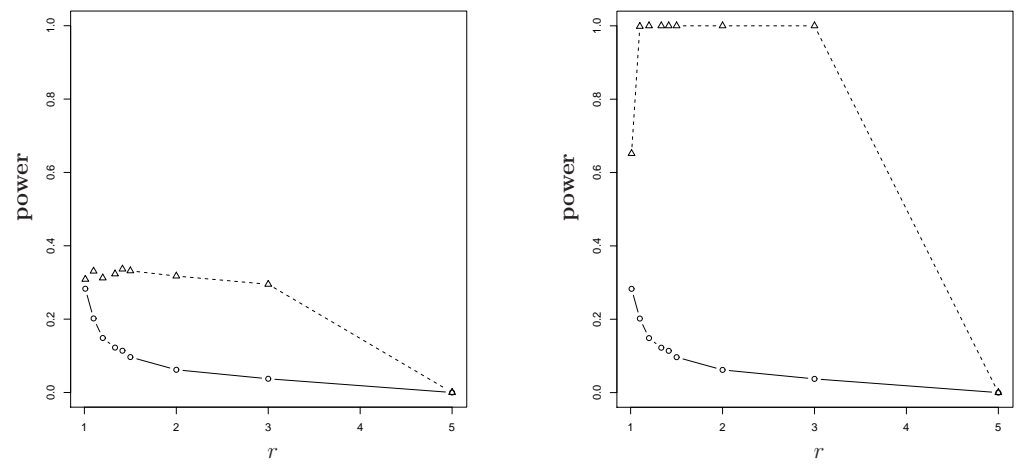

Figure 6: Monte Carlo power using the asymptotic critical value against segregation alternatives $H_{\sqrt{3} / 8}^{S}$ (left) and $H_{\sqrt{3} / 4}^{S}$ (right) as a function of $r$, for $n=10$. The circles represent the empirical significance levels while triangles represent the empirical power values. The $r$ values plotted are $1,11 / 10,12 / 10,4 / 3, \sqrt{2},, 2,3,5,10$.
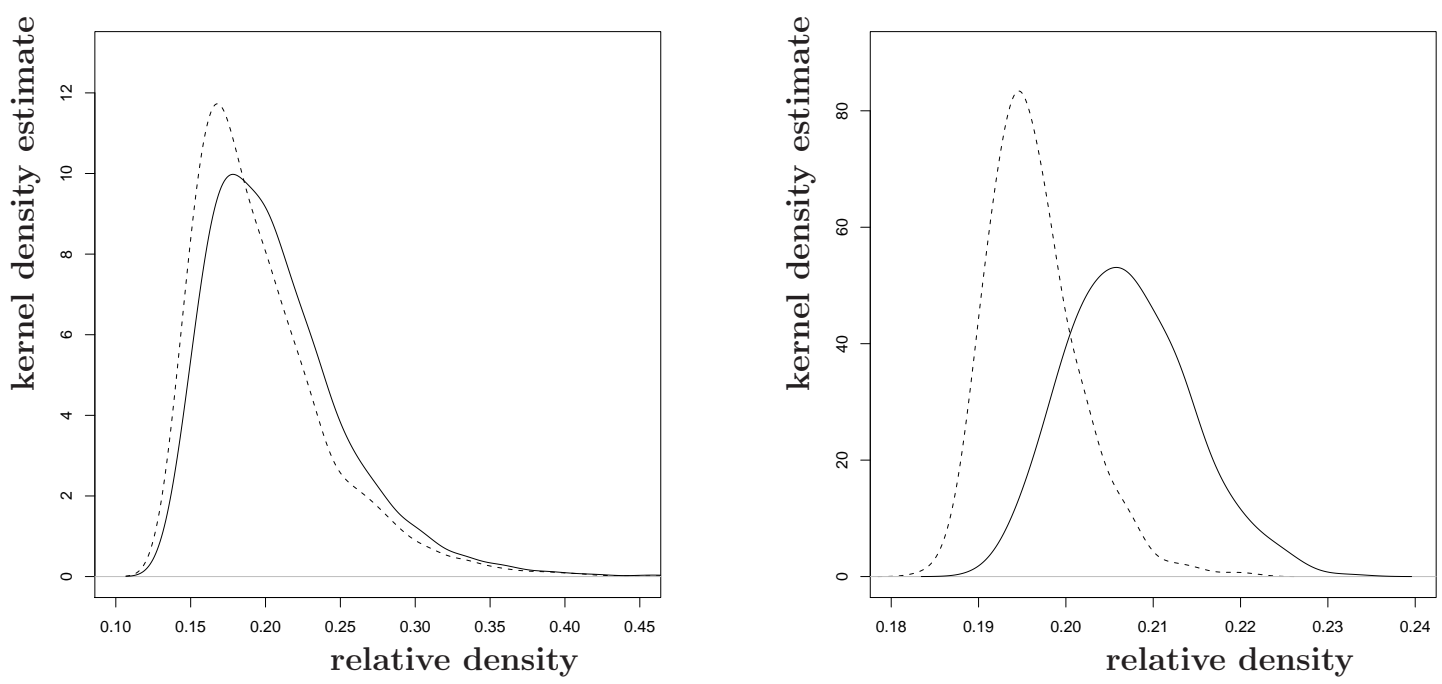

Figure 7: Two Monte Carlo experiments against the association alternative $H_{\sqrt{3} / 12}^{A}$. Depicted are kernel density estimates for $\rho_{n}(11 / 10)$ for $n=10$ (left) and $n=100$ (right) under the null (solid) and alternative (dashed). 

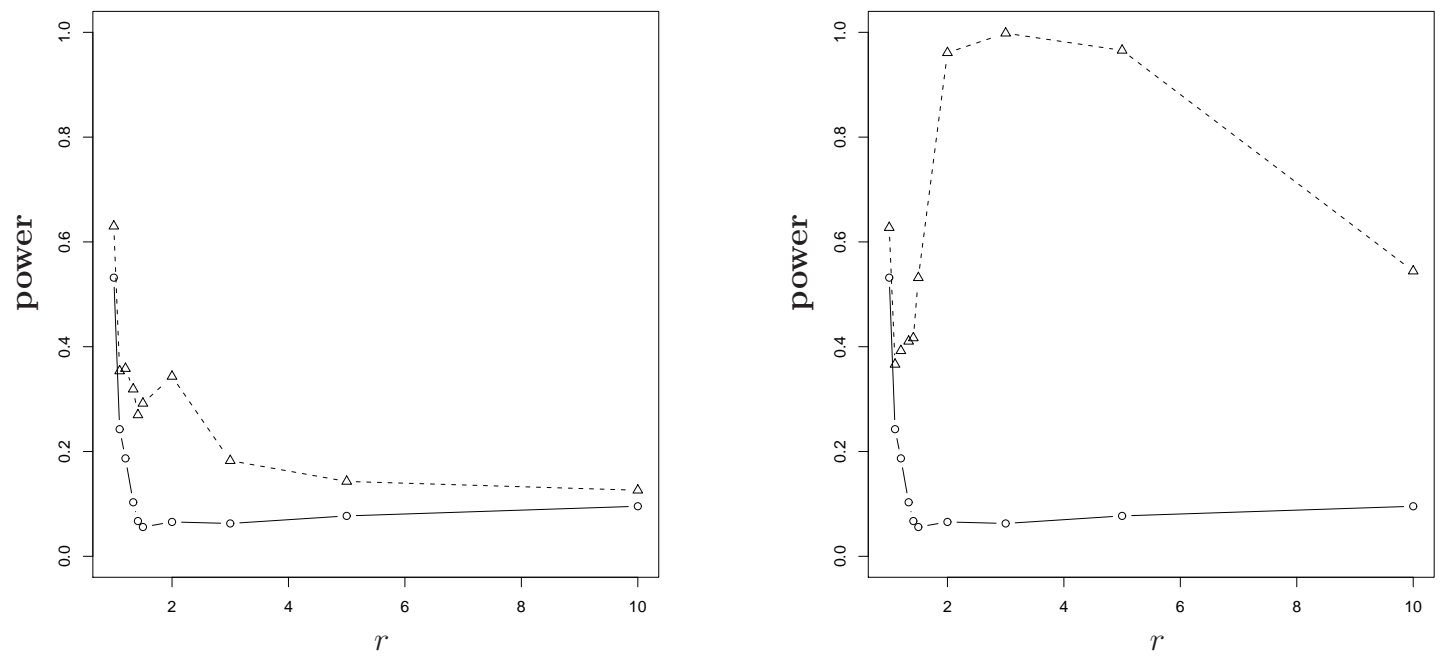

Figure 8: Monte Carlo power using the asymptotic critical value against association alternatives $H_{\sqrt{3} / 12}^{A}$ (left) and $H_{5 \sqrt{3} / 24}^{A}$ (right) as a function of $r$, for $n=10$. The $r$ values plotted are $1,11 / 10,12 / 10,4 / 3, \sqrt{2}, 2,3,5,10$.

segregation alternative $H_{\epsilon}^{S}$ and association alternative $H_{\epsilon}^{A}$, the PAE of $\rho_{n}(r)$ is given by

$$
\operatorname{PAE}^{S}(r)=\frac{\left(\mu_{S}^{\prime \prime}(r, \epsilon=0)\right)^{2}}{\nu(r)} \text { and } \operatorname{PAE}^{A}(r)=\frac{\left(\mu_{A}^{\prime \prime}(r, \epsilon=0)\right)^{2}}{\nu(r)},
$$

respectively, since $\mu_{S}^{\prime}(r, \epsilon=0)=\mu_{A}^{\prime}(r, \epsilon=0)=0$. Equation (9) provides the denominator; the numerator requires $\mu(r, \epsilon)$ which is provided in Appendix 2 for under both segregation and association alternatives, where we only use the intervals of $r$ that donot vanish as $\epsilon \rightarrow 0$.

In Figure 9 we present the PAE as a function of $r$ for both segregation and association. Notice that $\operatorname{PAE}^{S}(r=1)=160 / 7 \approx 22.8571, \lim _{r \rightarrow \infty} \operatorname{PAE}^{S}(r)=\infty, \operatorname{PAE}^{A}(r=1)=174240 / 17 \approx 10249.4118$, $\lim _{r \rightarrow \infty} \operatorname{PAE}^{A}(r)=0, \operatorname{argsup}_{r \in[1, \infty)} \mathrm{PAE}^{A}(r) \approx 1.006$ with $\sup _{r \in[1, \infty)} \mathrm{PAE}^{A}(r) \approx 10399.7726 . \mathrm{PAE}^{A}(r)$ has also a local supremum at $r_{l} \approx 1.4356$ with $\mathrm{PAE}^{A}\left(r_{l}\right) \approx 3630.8932$. Based on the asymptotic efficiency analysis, we suggest, for large $n$ and small $\epsilon$, choosing $r$ large for testing against segregation and choosing $r$ small for testing against association.

\subsection{Hodges-Lehmann Asymptotic Efficacy}

Hodges-Lehmann asymptotic efficiency (HLAE) of $\rho_{n}(r)$ (see e.g. Hodges and Lehmann (1956)) under $H_{\epsilon}^{S}$ is given by

$$
\operatorname{HLAE}^{S}(r, \epsilon):=\frac{\left(\mu_{S}(r, \epsilon)-\mu(r)\right)^{2}}{\nu_{S}(r, \epsilon)} .
$$

HLAE for association is defined similarly. Unlike PAE, HLAE does not involve the limit as $\epsilon \rightarrow 0$. Since this requires the mean and, especially, the asymptotic variance of $\rho_{n}(r)$ under an alternative, we investigate HLAE for specific values of $\epsilon$. Figure 10 contains a graph of HLAE against segregation as a function of $r$ for $\epsilon=\sqrt{3} / 8, \sqrt{3} / 4,2 \sqrt{3} / 7$. See Appendix 3 for explicit forms of $\mu_{S}(r, \epsilon)$ and $\nu_{S}(r, \epsilon)$ for $\epsilon=\sqrt{3} / 4$.

From Figure 10, we see that, against $H_{\epsilon}^{S}, \operatorname{HLAE}^{S}(r, \epsilon)$ appears to be an increasing function, dependent on $\epsilon, \overline{\text { of }} r$. Let $r_{d}(\epsilon)$ be the minimum $r$ such that $\bar{\rho}_{n}(r)$ becomes degenerate under the alternative $H_{\epsilon}^{S}$. Then 

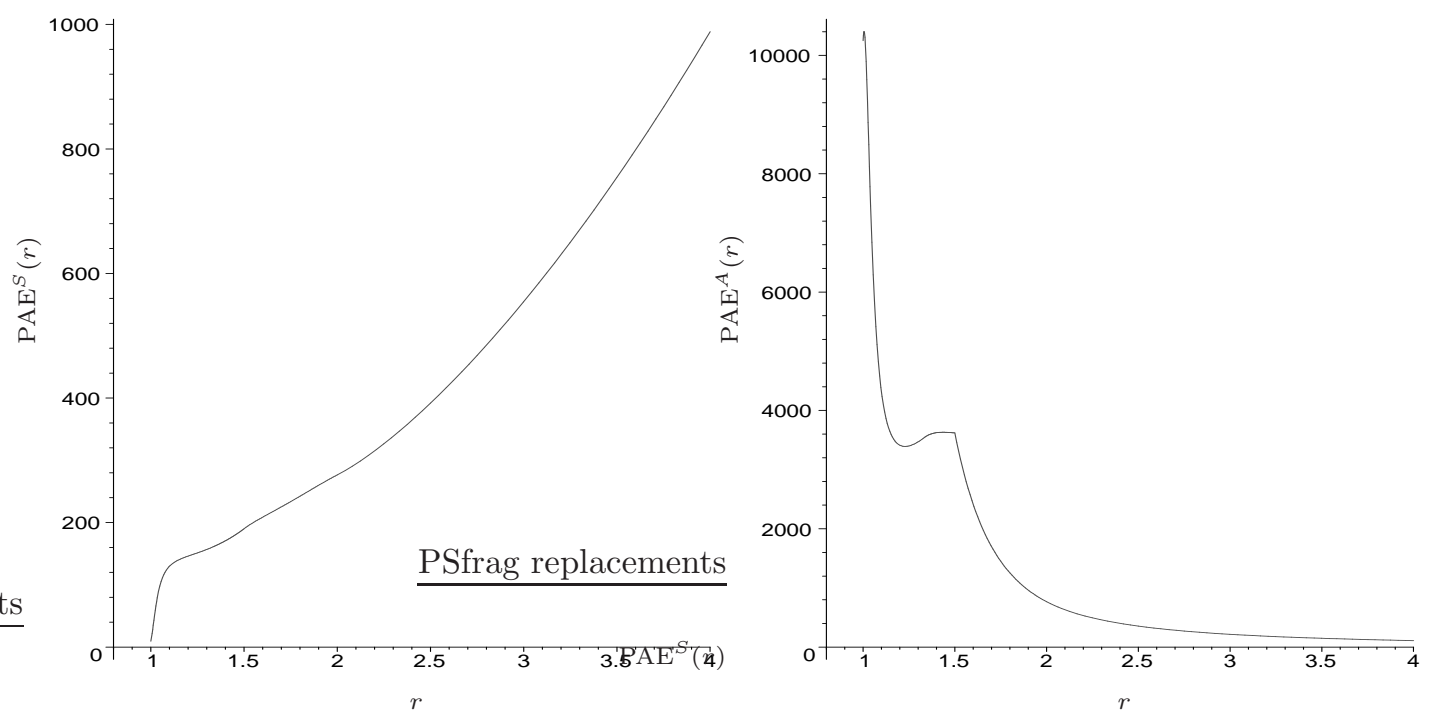

Figure 9: Pitman asymptotic efficiency against segregation (left) and association (right) as a function of $r$. Notice that vertical axes are differently scaled.
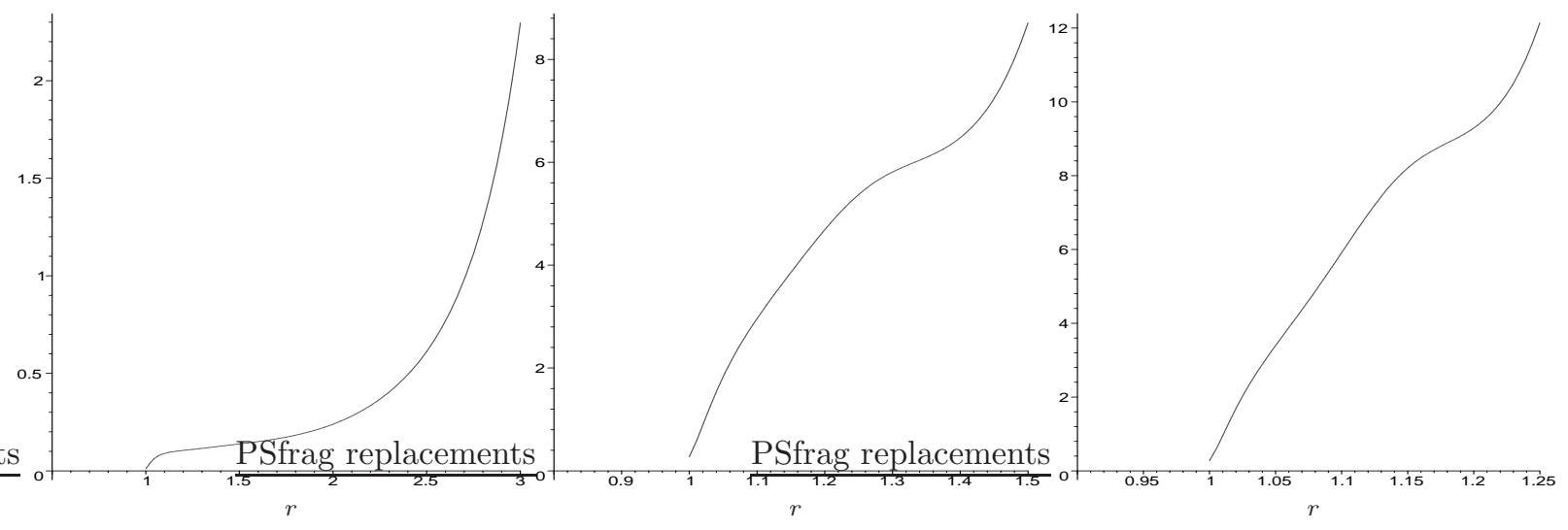

Figure 10: Hodges-Lehmann asymptotic efficiency against segregation alternative $H_{\epsilon}^{S}$ as a function of $r$ for $\epsilon=\sqrt{3} / 8, \sqrt{3} / 4,2 \sqrt{3} / 7$ (left to right).

$r_{d}(\sqrt{3} / 8)=4, r_{d}(\sqrt{3} / 4)=2$, and $r_{d}(2 \sqrt{3} / 7)=2$. In fact, for $\epsilon \in(0, \sqrt{3} / 4], r_{d}(\epsilon)=\sqrt{3} /(2 \epsilon)$ and for $\epsilon \in(\sqrt{3} / 4, \sqrt{3} / 3), r_{d}(\epsilon)=\sqrt{3} / \epsilon-2$. Notice that $\lim _{r \rightarrow r_{d}(\epsilon)} \operatorname{HLAE}^{S}(r, \epsilon)=\infty$, which is in agreement with $\mathrm{PAE}^{S}$ as $\epsilon \rightarrow 0$; since as $\epsilon \rightarrow 0$, HLAE becomes PAE and $r_{d}(\epsilon) \rightarrow \infty$ and under $H_{0}, \rho_{n}(r)$ is degenerate for $r=\infty$. So HLAE suggests choosing $r$ large against segregation, but in fact choosing $r$ too large will reduce power since $r \geq r_{d}(\epsilon)$ guarantees the complete digraph under the alternative and, as $r$ increases therefrom, provides an ever greater probability of seeing the complete digraph under the null.

Figure 11 contains a graph of HLAE against association as a function of $r$ for $\epsilon=5 \sqrt{3} / 24, \sqrt{3} / 12, \sqrt{3} / 21$. See Appendix 3 for explicit forms of $\mu_{A}(r, \epsilon)$ and $\nu_{A}(r, \epsilon)$ for $\epsilon=\sqrt{3} / 12$. Notice that since $\nu(r, \epsilon)=0$ for $\epsilon \geq \sqrt{3} / 12, \operatorname{HLAE}^{A}(r=1, \epsilon)=\infty$ for $\epsilon \geq \sqrt{3} / 12$ and $\lim _{r \rightarrow \infty} \operatorname{HLAE}^{A}(r, \epsilon)=0$.

In Figure 11 we see that, against $H_{\epsilon}^{A}, \operatorname{HLAE}^{A}(r, \epsilon)$ has a local supremum for $r$ sufficiently larger than 1. Let $\tilde{r}$ be the value at which this local supremum is attained. Then $\tilde{r}(5 \sqrt{3} / 24) \approx 3.2323, \tilde{r}(\sqrt{3} / 12) \approx 1.5676$, and $\tilde{r}(\sqrt{3} / 21) \approx 1.533$. Note that, as $\epsilon$ gets smaller, $\tilde{r}$ gets smaller. Furthermore, $\operatorname{HLAE}^{A}(r=1, \sqrt{3} / 21)<\infty$ and as $\epsilon \rightarrow 0, \tilde{r}$ becomes the global supremum, and $\operatorname{PAE}^{A}(r=1)=0$ and $\operatorname{argsup}_{r \geq 1} \operatorname{PAE}^{A}(r=1) \approx 1.006$. So, when testing against association, HLAE suggests choosing moderate $r$, whereas PAE suggests choosing small $r$. 

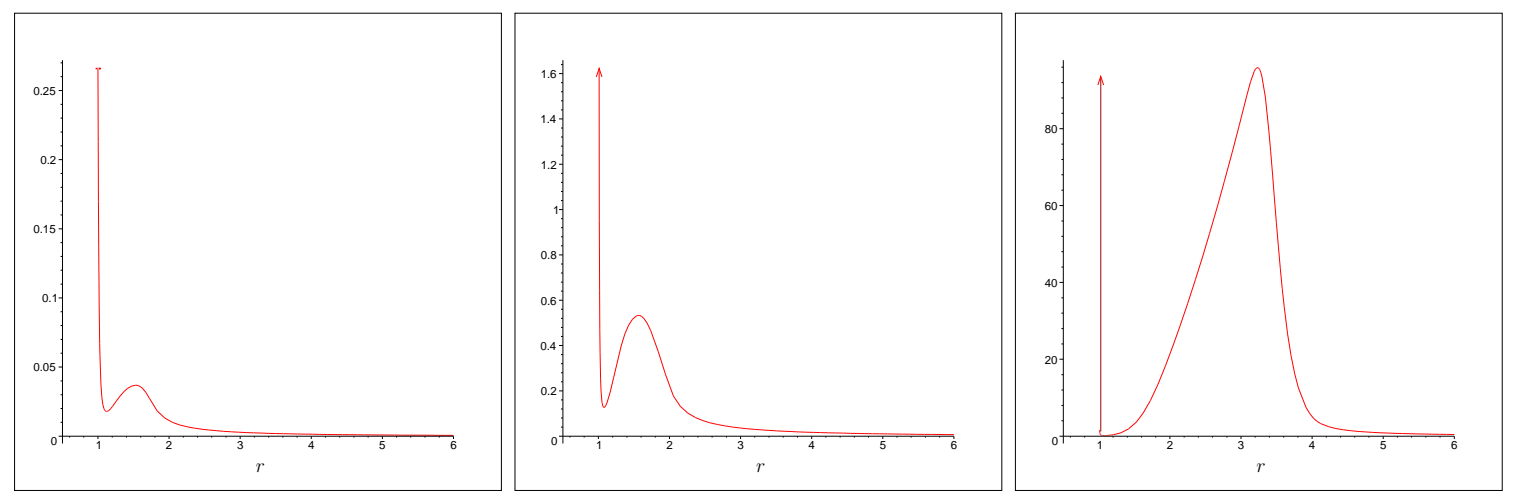

Figure 11: Hodges-Lehmann asymptotic efficiency against association alternative $H_{\epsilon}^{A}$ as a function of $r$ for $\epsilon=\sqrt{3} / 21, \sqrt{3} / 12,5 \sqrt{3} / 24$ (left to right).

\subsection{Asymptotic Power Function Analysis}

The asymptotic power function (see e.g. Kendall and Stuart (1979)) can also be investigated as a function of $r, n$, and $\epsilon$ using the asymptotic critical value and an appeal to normality. Under a specific segregation alternative $H_{\epsilon}^{S}$, the asymptotic power function is given by

$$
\Pi^{S}(r, n, \epsilon)=1-\Phi\left(\frac{z_{(1-\alpha)} \sqrt{\nu(r)}+\sqrt{n}\left(\mu(r)-\mu_{S}(r, \epsilon)\right)}{\sqrt{\nu_{S}(r, \epsilon)}}\right)
$$

where $z_{1-\alpha}=\Phi^{-1}(1-\alpha)$. Under $H_{\epsilon}^{A}$, we have

$$
\Pi^{A}(r, n, \epsilon)=\Phi\left(\frac{z_{\alpha} \sqrt{\nu(r)}+\sqrt{n}\left(\mu(r)-\mu_{A}(r, \epsilon)\right)}{\sqrt{\nu_{A}(r, \epsilon)}}\right) .
$$

Analysis of Figure 12 shows that, against $H_{\sqrt{3} / 8}^{S}$, a large choice of $r$ is warranted for $n=100$ but, for smaller sample size, a more moderate $r$ is recommended. Against $H_{\sqrt{3} / 12}^{A}$, a moderate choice of $r$ is recommended for both $n=10$ and $n=100$. This is in agreement with Monte Carlo investigations.
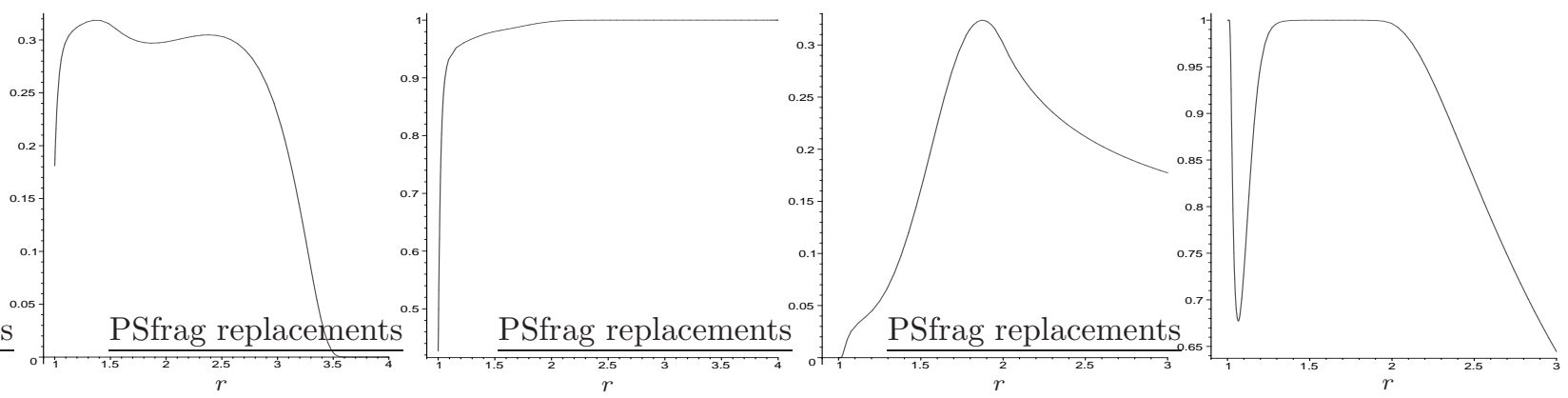

Figure 12: Asymptotic power function against segregation alternative $H_{\sqrt{3} / 8}^{S}$ as a function of $r$ for $n=10$ (first from left) and $n=100$ (second) and association alternative $H_{\sqrt{3} / 12}^{A}$ as a function of $r$ for $n=10$ (third) and $n=100$ (fourth). 


\section{Multiple Triangle Case}

Suppose $\mathcal{Y}$ is a finite collection of points in $\mathbb{R}^{2}$ with $|\mathcal{Y}| \geq 3$. Consider the Delaunay triangulation (assumed to exist) of $\mathcal{Y}$, where $T_{j}$ denotes the $j^{\text {th }}$ Delaunay triangle, $J$ denotes the number of triangles, and $C_{H}(\mathcal{Y})$ denotes the convex hull of $\mathcal{Y}$. We wish to test $H_{0}: X_{i} \stackrel{i i d}{\sim} \mathcal{U}\left(C_{H}(\mathcal{Y})\right)$ against segregation and association alternatives.

The digraph $D$ is constructed using $N_{\mathcal{Y}_{j}}^{r}(\cdot)$ as described in Section 2.3. where for $X_{i} \in T_{j}$ the three points in $\mathcal{Y}$ defining the Delaunay triangle $T_{j}$ are used as $\mathcal{Y}_{j}$. Let $\rho_{n}(r, J)$ be the relative density of the digraph based on $\mathcal{X}_{n}$ and $\mathcal{Y}$ which yields $J$ Delaunay triangles, and let $w_{j}:=A\left(T_{j}\right) / A\left(C_{H}(\mathcal{Y})\right.$ ) for $j=1, \ldots, J$, where $A\left(C_{H}(\mathcal{Y})\right)=\sum_{j=1}^{J} A\left(T_{j}\right)$ with $A(\cdot)$ being the area functional. Then we obtain the following as a corollary to Theorem 2 .

Corollary 1: The asymptotic null distribution for $\rho_{n}(r, J)$ conditional on $\mathcal{W}=\left\{w_{1}, \ldots, w_{J}\right\}$ for $r \in$ $[1, \infty]$ is given by $\mathcal{N}(\mu(r, J), \nu(r, J) / n)$ provided that $\nu(r, J)>0$ with

$$
\mu(r, J):=\mu(r) \sum_{j=1}^{J} w_{j}^{2}, \text { and } \nu(r, J):=\nu(r) \sum_{j=1}^{J} w_{j}^{3}+4 \mu(r)^{2}\left[\sum_{j=1}^{J} w_{j}^{3}-\left(\sum_{j=1}^{J} w_{j}^{2}\right)^{2}\right]
$$

where $\mu(r)$ and $\nu(r)$ are given by equations (8) and (9), respectively.

\section{Proof: See Appendix 4.}

By an appropriate application of Jensen's Inequality, we see that $\sum_{j=1}^{J} w_{j}^{3} \geq\left(\sum_{j=1}^{J} w_{j}^{2}\right)^{2}$. Therefore, $\nu(r, J)=0$ iff $\nu(r)=0$ and $\sum_{j=1}^{J} w_{j}^{3}=\left(\sum_{j=1}^{J} w_{j}^{2}\right)^{2}$, so asymptotic normality may hold even when $\nu(r)=0$.

Similarly, for the segregation (association) alternatives with $4 \epsilon^{2} / 3 \times 100 \%$ of the triangles around the vertices of each triangle is forbidden (allowed), we obtain the above asymptotic distribution of $\rho_{n}(r)$ with $\mu(r)$ being replaced by $\mu_{S}(r, \epsilon), \nu(r)$ by $\nu_{S}(r, \epsilon), \mu(r, J)$, by $\mu_{S}(r, J, \epsilon)$, and $\nu(r, J)$ by $\nu_{S}(r, J, \epsilon)$. Likewise for association.

Thus in the case of $J>1$, we have a (conditional) test of $H_{0}: X_{i} \stackrel{i i d}{\sim} \mathcal{U}\left(C_{H}(\mathcal{Y})\right)$ which once again rejects against segregation for large values of $\rho_{n}(r, J)$ and rejects against association for small values of $\rho_{n}(r, J)$.

Depicted in Figure 13 are the segregation (with $\delta=1 / 16$ i.e. $\epsilon=\sqrt{3} / 8$ ), null, and association (with $\delta=1 / 4$ i.e. $\epsilon=\sqrt{3} / 12$ ) realizations (from left to right) with $n=1000,|\mathcal{Y}|=10$, and $J=13$. For the null realization, the $p$-value is greater than 0.1 for all $r$ values and both alternatives. For the segregation realization, we obtain $p<0.0031$ for $1<r \leq 5$ and $p>0.24$ for $r=1$ and $r \geq 10$. For the association realization, we obtain $p<0.0135$ for $1<r \leq 3, p=.14$ for $r=1$, and $p>0.25$ for for $r \geq 5$. Note that this is only for one realization of $\mathcal{X}_{n}$.

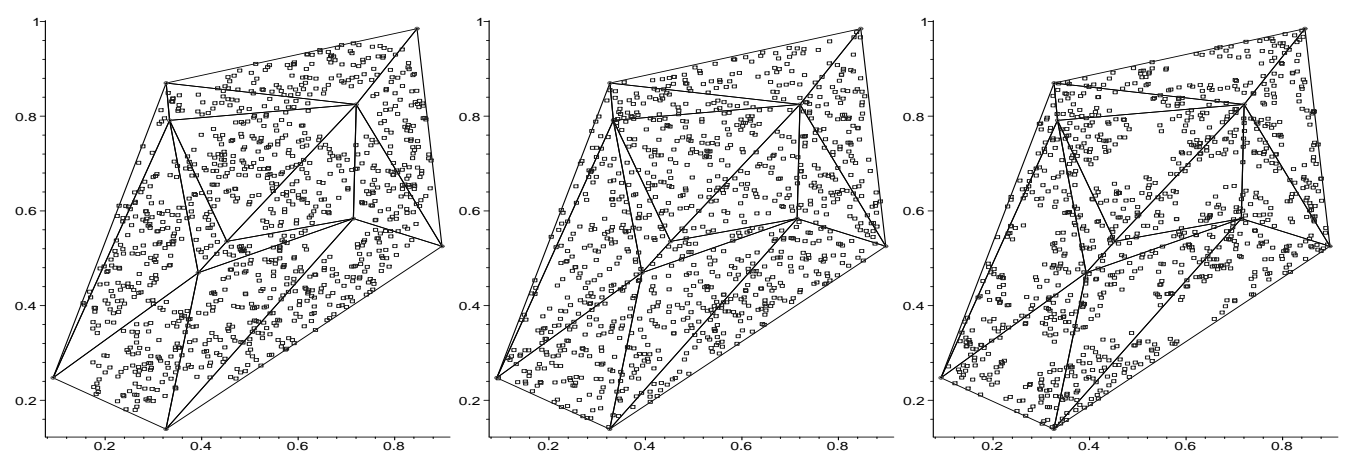

Figure 13: Realization of segregation (left), $H_{0}$ (middle), and association (right) for $|\mathcal{Y}|=10, J=13$, and $n=1000$. 

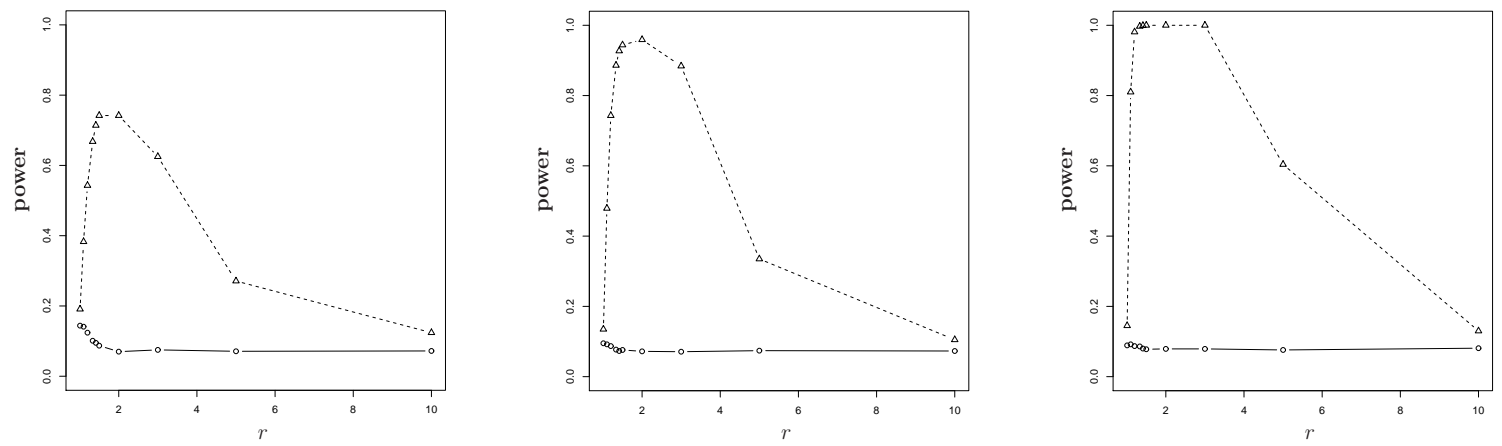

Figure 14: Monte Carlo power using the asymptotic critical value against $H_{\sqrt{3} / 8}^{S}$, as a function of $r$, for $n=100$ (left), $n=200$ (middle), and $n=500$ (right) conditional on the realization of $\mathcal{Y}$ in Figure 13 The circles represent the empirical significance levels while triangles represent the empirical power values.
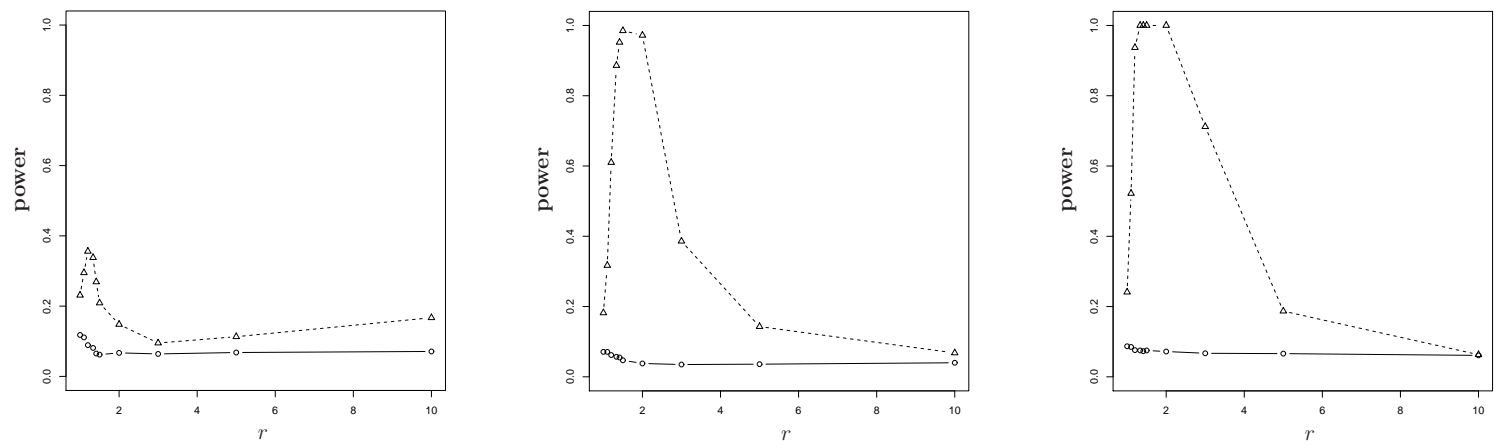

Figure 15: Monte Carlo power using the asymptotic critical value against $H_{\sqrt{3} / 12}^{A}$ as a function of $r$, for $n=100$ (left), $n=200$ (middle), and $n=500$ (right) conditional on the realization of $\mathcal{Y}$ in Figure 13 . The circles represent the empirical significance levels while triangles represent the empirical power values.

We implement the above described Monte Carlo experiment 1000 times with $n=100, n=200$, and $n=$ 500 and find the empirical significance levels $\widehat{\alpha}_{S}(n, J)$ and $\widehat{\alpha}_{A}(n, J)$ and the empirical powers $\widehat{\beta}_{n}^{S}(r, \sqrt{3} / 8, J)$ and $\widehat{\beta}_{n}^{A}(r, \sqrt{3} / 12, J)$. These empirical estimates are presented in Table 1 and plotted in Figures 14 and 15 Notice that the empirical significance levels are all larger than .05 for both alternatives, so this test is liberal in rejecting $H_{0}$ against both alternatives for the given realization of $\mathcal{Y}$ and $n$ values. The smallest empirical significance levels and highest empirical power estimates occur at moderate $r$ values $(r=3 / 2,2,3)$ against segregation and at smaller $r$ values $(r=\sqrt{2}, 3 / 2)$ against association. Based on this analysis, for the given realization of $\mathcal{Y}$, we suggest the use of moderate $r$ values for segregation and slightly smaller for association. Notice also that as $n$ increases, the empirical power estimates gets larger for both alternatives.

The conditional test presented here is appropriate when the $\mathcal{W}$ are fixed, not random. An unconditional version requires the joint distribution of the number and relative size of Delaunay triangles when $\mathcal{Y}$ is, for instance, a Poisson point pattern. Alas, this joint distribution is not available Okabe et al. (2000).

\subsection{Related Test Statistics in Multiple Triangle Case}

For $J>1$, we have derived the asymptotic distribution of $\rho_{n}(r, J)=|\mathcal{A}| /(n(n-1))$. Let $\mathcal{A}_{j}$ be the number of arcs, $n_{j}:=\left|\mathcal{X}_{n} \cap T_{j}\right|$, and $\rho_{n_{j}}(r)$ be the arc density for triangle $T_{j}$ for $j=1, \ldots, J$. So $\sum_{j=1}^{J} \frac{\bar{n}_{j}\left(n_{j}-1\right)}{n(n-1)} \rho_{n_{j}}(r)=$ 


\begin{tabular}{|c|c|c|c|c|c|c|c|c|c|c|}
\hline$r$ & 1 & $11 / 10$ & $6 / 5$ & $4 / 3$ & $\sqrt{2}$ & $3 / 2$ & 2 & 3 & 5 & 10 \\
\hline \multicolumn{11}{|c|}{$n=100, N=1000$} \\
\hline$\widehat{\alpha}_{S}(n, J)$ & 0.144 & 0.141 & 0.124 & 0.101 & 0.095 & 0.087 & 0.070 & 0.075 & 0.071 & 0.072 \\
\hline$\widehat{\beta}_{n}^{S}(r, \sqrt{3} / 8, J)$ & 0.191 & 0.383 & 0.543 & 0.668 & 0.714 & 0.742 & 0.742 & 0.625 & 0.271 & 0.124 \\
\hline$\widehat{\widehat{\alpha}}_{A}(n, J)$ & 0.118 & 0.111 & 0.089 & 0.081 & 0.065 & 0.062 & 0.067 & 0.064 & 0.068 & 0.071 \\
\hline$\widehat{\beta_{n}^{A}(r, \sqrt{3} / 12, J)}$ & 0.231 & 0.295 & 0.356 & 0.338 & 0.269 & 0.209 & 0.148 & 0.095 & 0.113 & 0.167 \\
\hline \multicolumn{11}{|c|}{$n=200, N=1000$} \\
\hline$\widehat{\alpha}_{S}(n, J)$ & 0.095 & 0.092 & 0.087 & 0.077 & 0.073 & 0.076 & 0.072 & 0.071 & 0.074 & 0.073 \\
\hline$\widehat{\beta}_{n}^{S}(r, \sqrt{3} / 8, J)$ & 0.135 & 0.479 & 0.743 & 0.886 & 0.927 & 0.944 & 0.959 & 0.884 & 0.335 & 0.105 \\
\hline$\widehat{\alpha}_{A}(n, J)$ & 0.071 & 0.071 & 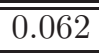 & 0.057 & 0.055 & 0.047 & 0.038 & 0.035 & 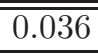 & 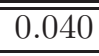 \\
\hline$\widehat{\beta}_{n}^{A}(r, \sqrt{3} / 12, J)$ & 0.182 & 0.317 & 0.610 & 0.886 & 0.952 & 0.985 & 0.972 & 0.386 & 0.143 & 0.068 \\
\hline \multicolumn{11}{|c|}{$n=500, N=1000$} \\
\hline$\widehat{\alpha}_{S}(n, J)$ & 0.089 & 0.092 & 0.087 & 0.086 & 0.080 & 0.078 & 0.079 & 0.079 & 0.076 & 0.081 \\
\hline$\widehat{\beta}_{n}^{S}(r, \sqrt{3} / 8, J)$ & 0.145 & 0.810 & 0.981 & 0.997 & 0.999 & 1.000 & 1.000 & 1.000 & 0.604 & 0.130 \\
\hline$\widehat{\alpha}_{A}(n, J)$ & 0.087 & 0.085 & 0.076 & 0.075 & 0.073 & 0.075 & 0.072 & 0.067 & 0.066 & 0.061 \\
\hline$\widehat{\beta}_{n}^{A}(r, \sqrt{3} / 12, J)$ & 0.241 & 0.522 & 0.937 & 1.000 & 1.000 & 1.000 & 1.000 & 0.712 & 0.187 & 0.063 \\
\hline
\end{tabular}

Table 1: The empirical significance level and empirical power values under $H_{\sqrt{3} / 8}^{S}$ and $H_{\sqrt{3} / 12}^{A}, N=1000$, $n=100$, and $J=13$, at $\alpha=.05$ for the realization of $\mathcal{Y}$ in Figure 13 .

$\rho_{n}(r, J)$, since $\sum_{j=1}^{J} \frac{n_{j}\left(n_{j}-1\right)}{n(n-1)} \rho_{n_{j}}(r)=\frac{\sum_{j=1}^{J}\left|\mathcal{A}_{j}\right|}{n(n-1)}=\frac{|\mathcal{A}|}{n(n-1)}=\rho_{n}(r, J)$.

Let $\widehat{U}_{n}:=\sum_{j=1}^{J} w_{j}^{2} \rho_{n_{j}}(r)$ where $w_{j}=A\left(T_{j}\right) / A\left(C_{H}(\mathcal{Y})\right)$. Since $\rho_{n_{j}}(r)$ are asymptotically independent, $\sqrt{n}\left(\widehat{U}_{n}-\mu(r, J)\right)$ and $\sqrt{n}\left(\rho_{n}(r, J)-\mu(r, J)\right)$ both converge in distribution to $\mathcal{N}(0, \nu(r, J))$.

In the denominator of $\rho_{n}(r, J)$, we use $n(n-1)$ as the maximum number of arcs possible. However, by definition, we can at most have a digraph with $J$ complete symmetric components of order $n_{j}$, for $j=1, \ldots, J$. Then the maximum number possible is $n_{t}:=\sum_{j=1}^{J} n_{j}\left(n_{j}-1\right)$. Then the (adjusted) arc density is $\rho_{n, J}^{a d j}:=\frac{|\mathcal{A}|}{n_{t}}$. Then $\rho_{n, J}^{a d j}(r)=\frac{\sum_{j=1}^{J}\left|\mathcal{A}_{j}\right|}{n_{t}}=\sum_{j=1}^{J} \frac{n_{j}\left(n_{j}-1\right)}{n_{t}} \rho_{n_{j}}(r)$. Since $\frac{n_{j}\left(n_{j}-1\right)}{n_{t}} \geq 0$ for each $j$, and $\sum_{j=1}^{J} \frac{n_{j}\left(n_{j}-1\right)}{n_{t}}=1, \rho_{n, J}^{a d j}(r)$ is a mixture of $\rho_{n_{j}}(r)$ 's. Then $\rho_{n, J}^{a d j}(r)$ is asymptotically normal with mean $\mathbf{E}\left[\rho_{n, J}^{a d j}(r)\right]=\mu(r, J)$ and the variance of $\rho_{n, J}^{a d j}(r)$ is

$$
\frac{1}{n}\left[\nu(r)\left(\sum_{j=1}^{J} w_{j}^{3} /\left(\sum_{j=1}^{J} w_{j}^{2}\right)^{2}\right)+4 \mu(r)^{2}\left(\sum_{j=1}^{J} w_{j}^{3} /\left(\sum_{j=1}^{J} w_{j}^{2}\right)^{2}-1\right)\right] .
$$

\subsection{Asymptotic Efficacy Analysis for $J>1$}

The PAE, HLAE, and asymptotic power function analysis are given for $J=1$ in Sections 4.3, 4.4, and 4.5 respectively. For $J>1$, the analysis will depend on both the number of triangles as well as the size of the triangles. So the optimal $r$ values with respect to these efficiency criteria for $J=1$ do not necessarily hold for $J>1$, hence the analyses need to be updated, given the values of $J$ and $\mathcal{W}$.

Under segregation alternative $H_{\epsilon}^{S}$, the PAE of $\rho_{n}(r, J)$ is given by

$$
\operatorname{PAE}_{J}^{S}(r)=\frac{\left(\mu_{S}^{\prime \prime}(r, J, \epsilon=0)\right)^{2}}{\nu(r, J)}=\frac{\left(\mu_{S}^{\prime \prime}(r, \epsilon=0) \sum_{j=1}^{J} w_{j}^{2}\right)^{2}}{\nu(r) \sum_{j=1}^{J} w_{j}^{3}+4 \mu_{S}(r, \epsilon=0)^{2}\left(\sum_{j=1}^{J} w_{j}^{3}-\left(\sum_{j=1}^{J} w_{j}^{2}\right)^{2}\right)}
$$

Under association alternative $H_{\epsilon}^{A}$ the PAE of $\rho_{n}(r, J)$ is similar. In Figure [16 we present the PAE as a 

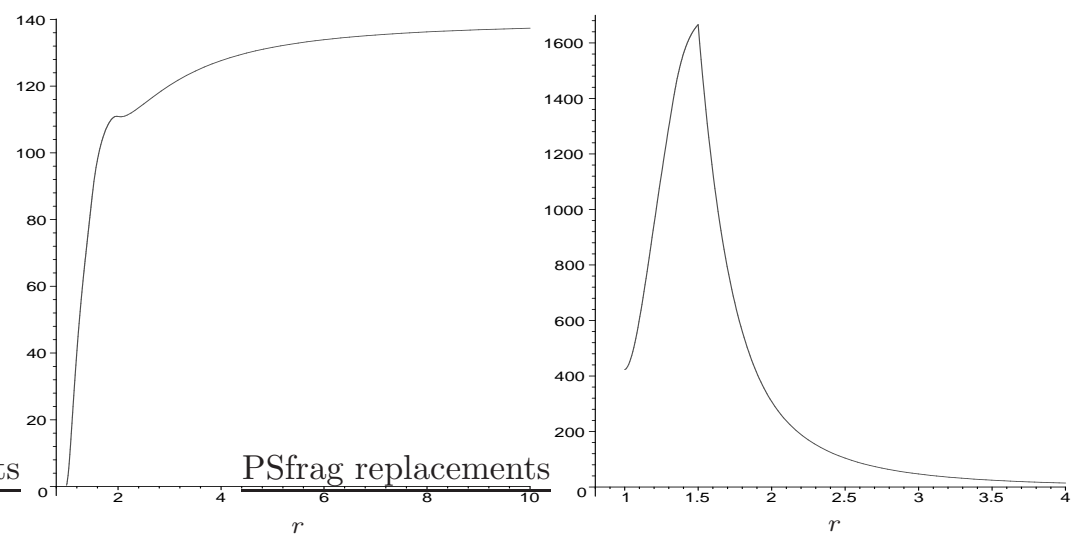

Figure 16: Pitman asymptotic efficiency against segregation (left) and association (right) as a function of $r$ with $J=13$. Notice that vertical axes are differently scaled.
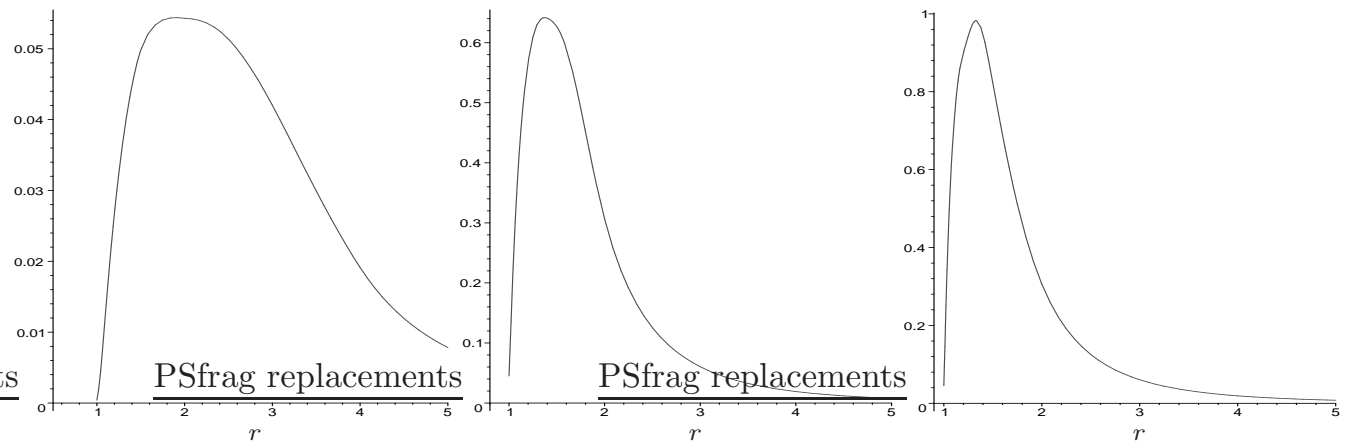

Figure 17: Hodges-Lehmann asymptotic efficiency against segregation alternative $H_{\epsilon}^{S}$ as a function of $r$ for $\epsilon=\sqrt{3} / 8, \sqrt{3} / 4,2 \sqrt{3} / 7$ (left to right) and $J=13$.

function of $r$ for both segregation and association conditional on the realization of $\mathcal{Y}$ in Figure 13 . Notice that, unlike $J=1$ case, $\operatorname{PAE}_{J}^{S}(r)$ is bounded. Some values of note are $\operatorname{PAE}_{J}^{S}\left(\rho_{n}(1)\right)=.3884, \lim _{r \rightarrow \infty} \operatorname{PAE}_{J}^{S}(r)=$ $\frac{8 \sum_{j=1}^{J} w_{j}^{2}}{256\left(\sum_{j=1}^{J} w_{j}^{3}-\left(\sum_{j=1}^{J} w_{j}^{2}\right)^{2}\right)} \approx 139.34, \operatorname{argsup}_{r \in[1,2]} \operatorname{PAE}_{J}^{S}(r) \approx 1.974$. As for association, $\operatorname{PAE}_{J}^{A}(r=1)=$ 422.9551, $\lim _{r \rightarrow \infty} \operatorname{PAE}_{J}^{A}(r)=0, \operatorname{argsup}_{r>1} \operatorname{PAE}_{J}^{A}(r)=1.5$ with $\operatorname{PAE}_{J}^{A}(r=1.5) \approx 1855.9672$. Based on the asymptotic efficiency analysis, we suggest, for large $n$ and small $\epsilon$, choosing moderate $r$ for testing against segregation and association.

Under segregation, the HLAE of $\rho_{n}(r, J)$ is given by

$$
\operatorname{HLAE}_{J}^{S}(r, \epsilon):=\frac{\left(\mu_{S}(r, J, \epsilon)-\mu(r, J)\right)^{2}}{\nu_{S}(r, J, \epsilon)}=\frac{\left(\mu_{S}(r, \epsilon)\left(\sum_{j=1}^{J} w_{j}^{2}\right)-\mu(r)\left(\sum_{j=1}^{J} w_{j}^{2}\right)\right)^{2}}{\nu_{S}(r, \epsilon) \sum_{j=1}^{J} w_{j}^{3}+4 \mu_{S}(r, \epsilon)^{2}\left(\sum_{j=1}^{J} w_{j}^{3}-\left(\sum_{j=1}^{J} w_{j}^{2}\right)^{2}\right)} .
$$

Notice that $\operatorname{HLAE}_{J}^{S}(r, \epsilon=0)=0$ and $\lim _{\rightarrow \infty} \operatorname{HLAE}_{J}^{S}(r, \epsilon)=0$ and HLAE is bounded provided that $\nu(r, J)>$ 0 .

We calculate HLAE of $\rho_{n}(r, J)$ under $H_{\epsilon}^{S}$ for $\epsilon=\sqrt{3} / 8, \epsilon=\sqrt{3} / 4$, and $\epsilon=2 \sqrt{3} / 7$. In Figure 17 we present $\operatorname{HLAE}_{J}^{S}(r, \epsilon)$ for these $\epsilon$ values conditional on the realization of $\mathcal{Y}$ in Figure 13 , Note that with $\epsilon=\sqrt{3} / 8, \operatorname{HLAE}_{J}^{S}(r=1, \sqrt{3} / 8) \approx .0004$ and $\operatorname{argsup}_{r \in[1, \infty]} \operatorname{HLAE}_{J}^{S}(r, \sqrt{3} / 8) \approx 1.8928$ with the supremum $\approx$ .0544. With $\epsilon=\sqrt{3} / 4, \operatorname{HLAE}_{J}^{S}(r=1, \sqrt{3} / 4) \approx .0450$ and $\operatorname{argsup}_{r \in[1, \infty]} \operatorname{HLAE}_{J}^{S}(r, \sqrt{3} / 4) \approx 1.3746$ with the supremum $\approx .6416$. With $\epsilon=2 \sqrt{3} / 7, \operatorname{HLAE}_{J}^{S}(r=1,2 \sqrt{3} / 7) \approx .045$ and $\operatorname{argsup}_{r \in[1, \infty]} \operatorname{HLAE}_{J}^{S}(r, 2 \sqrt{3} / 7) \approx$ 1.3288 with the supremum $\approx .9844$. Furthermore, we observe that $\operatorname{HLAE}_{J}^{S}(r, 2 \sqrt{3} / 7)>\operatorname{HLAE}_{J}^{S}(r, \sqrt{3} / 4)>$ 
$\operatorname{HLAE}_{J}^{S}(r, \sqrt{3} / 8)$. Based on the HLAE analysis for the given $\mathcal{Y}$ we suggest moderate $r$ values for moderate segregation and small $r$ values for severe segregation.

The explicit form of $\operatorname{HLAE}_{J}^{A}(r, \epsilon)$ is similar to $\operatorname{HLAE}_{J}^{S}(r, \epsilon)$ which implies $\operatorname{HLAE}_{J}^{A}(r, \epsilon=0)=0$ and $\lim _{\rightarrow \infty} \operatorname{HLAE}_{J}^{A}(r, \epsilon)=0$.

We calculate HLAE of $\rho_{n}(r, J)$ under $H_{\epsilon}^{A}$ for $\epsilon=\sqrt{3} / 21, \epsilon=\sqrt{3} / 12$, and $\epsilon=5 \sqrt{3} / 24$. In Figure 18 we present $\operatorname{HLAE}_{J}^{S}(r, \epsilon)$ for these $\epsilon$ values conditional on the realization of $\mathcal{Y}$ in Figure 13 Note that with $\epsilon=$
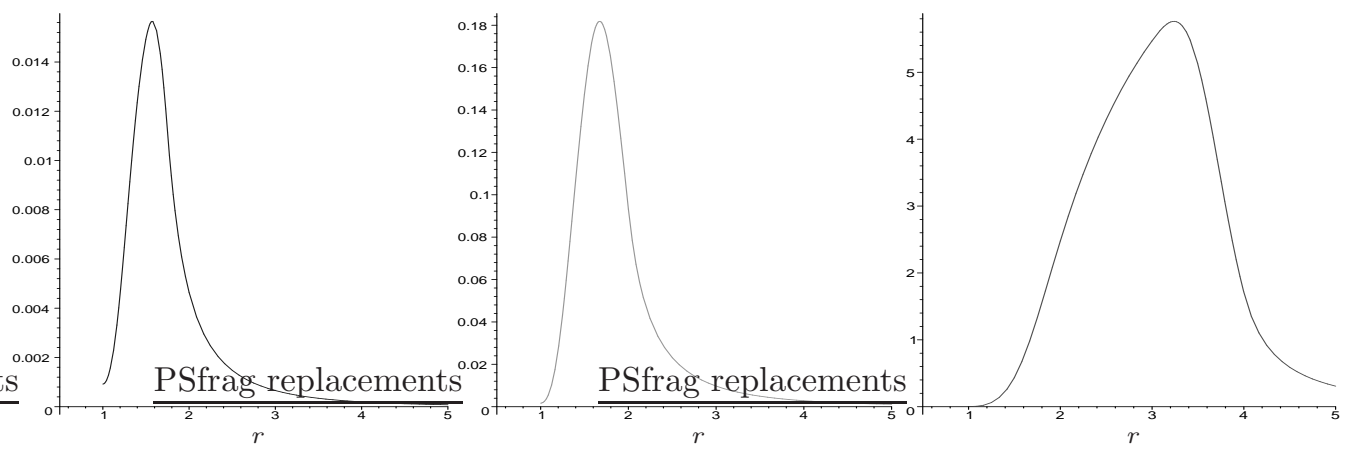

Figure 18: Hodges-Lehmann asymptotic efficiency against association alternative $H_{\epsilon}^{A}$ as a function of $r$ for $\epsilon=\sqrt{3} / 21, \sqrt{3} / 12,5 \sqrt{3} / 24$ (left to right) and $J=13$.

$\sqrt{3} / 21, \operatorname{HLAE}_{J}^{A}(r=1, \sqrt{3} / 21) \approx .0009$ and $\operatorname{argsup}_{r \in[1, \infty]} \operatorname{HLAE}_{J}^{A}(r, \sqrt{3} / 21) \approx 1.5734$ with the supremum $\approx .0157$. With $\epsilon=\sqrt{3} / 12, \operatorname{HLAE}_{J}^{A}(r=1, \sqrt{3} / 12) \approx .0168$ and $\operatorname{argsup}_{r \in[1, \infty]} \operatorname{HLAE}_{J}^{A}(r, \sqrt{3} / 12) \approx 1.6732$ with the supremum $\approx .1818$. With $\epsilon=5 \sqrt{3} / 24, \operatorname{HLAE}_{J}^{A}(r=1,5 \sqrt{3} / 24) \approx .0017$ and

$\operatorname{argsup}_{r \in[1, \infty]} \operatorname{HLAE}_{J}^{A}(r, 5 \sqrt{3} / 24) \approx 3.2396$ with the supremum $\approx 5.7616$. Furthermore, we observe that $\operatorname{HLAE}_{J}^{A}(r, 5 \sqrt{3} / 24)>\operatorname{HLAE}_{J}^{A}(r, \sqrt{3} / 12)>\operatorname{HLAE}_{J}^{A}(r, \sqrt{3} / 21)$. Based on the HLAE analysis for the given $\mathcal{Y}$ we suggest moderate $r$ values for moderate association and large $r$ values for severe association.

\section{Discussion and Conclusions}

In this article we investigate the mathematical properties of a random digraph method for the analysis of spatial point patterns.

The first proximity map similar to the $r$-factor proximity map $N_{\mathcal{Y}}^{r}$ in literature is the spherical proximity map $N_{S}(x):=B(x, r(x))$, (see the references for CCCD in the Introduction). A slight variation of $N_{S}$ is the arc-slice proximity map $N_{A S}(x):=B(x, r(x)) \cap T(x)$ where $T(x)$ is the Delaunay cell that contains $x$ (see Cevhan and Priebe (2003a)). Furthermore, Ceyhan and Priebe introduced the central similarity proximity map $N_{C S}$ in Cevhan and Priebe (2003a) and $N_{\mathcal{Y}}^{r}$ in Cevhan and Priebe (2003b). The $r$-factor proximity map, when compared to the others, has the advantages that the asymptotic distribution of the domination number $\gamma_{n}\left(N_{\mathcal{Y}}^{r}\right)$ is tractable (see Ceyhan and Priebe (2003b)), an exact minimum dominating set can be found in polynomial time. Moreover $N_{\mathcal{Y}}^{r}$ and $N_{C S}$ are geometry invariant for uniform data over triangles. Additionally, the mean and variance of relative density $\rho_{n}$ is not analytically tractable for $N_{S}$ and $N_{A S}$. While $N_{\mathcal{Y}}^{r}(x), N_{C S}(x)$, and $N_{A S}(x)$ are well defined only for $x \in C_{H}(\mathcal{Y})$, the convex hull of $\mathcal{Y}, N_{S}(x)$ is well defined for all $x \in \mathbb{R}^{d}$. The proximity maps $N_{S}$ and $N_{A S}$ require no effort to extend to higher dimensions.

The $N_{S}$ (the proximity map associated with CCCD) is used in classification in the literature, but not for testing spatial patterns between two or more classes. We develop a technique to test the patterns of segregation or association. There are many tests available for segregation and association in ecology literature. See Dixon (1994) for a survey on these tests and relevant references. Two of the most commonly used tests are Pielou's $\chi^{2}$ test of independence and Ripley's test based on $K(t)$ and $L(t)$ functions. However, the test we introduce here is not comparable to either of them. Our test is a conditional test - conditional on 
a realization of $J$ (number of Delaunay triangles) and $\mathcal{W}$ (the set of relative areas of the Delaunay triangles) and we require the number of triangles $J$ is fixed and relatively small compared to $n=\left|\mathcal{X}_{n}\right|$. Furthermore, our method deals with a slightly different type of data than most methods to examine spatial patterns. The sample size for one type of point (type $\mathcal{X}$ points) is much larger compared to the the other (type $\mathcal{Y}$ points). This implies that in practice, $\mathcal{Y}$ could be stationary or have much longer life span than members of $\mathcal{X}$. For example, a special type of fungi might constitute $\mathcal{X}$ points, while the tree species around which the fungi grow might be viewed as the $\mathcal{Y}$ points.

There are two major types of asymptotic structures for spatial data Lahiri (1996). In the first, any two observations are required to be at least a fixed distance apart, hence as the number of observations increase, the region on which the process is observed eventually becomes unbounded. This type of sampling structure is called "increasing domain asymptotics". In the second type, the region of interest is a fixed bounded region and more or more points are observed in this region. Hence the minimum distance between data points tends to zero as the sample size tends to infinity. This type of structure is called "infill asymptotics", due to Cressie Cressie (1991). The sampling structure for our asymptotic analysis is infill, as only the size of the type $X$ process tends to infinity, while the support, the convex hull of a given set of points from type $Y$ process, $C_{H}(\mathcal{Y})$ is a fixed bounded region.

Moreover, our statistic that can be written as a $U$-statistic based on the locations of type $X$ points with respect to type $Y$ points. This is one advantage of the proposed method: most statistics for spatial patterns can not be written as $U$-statistics. The $U$-statistic form avails us the asymptotic normality, once the mean and variance is obtained by tedious detailed geometric calculations.

The null hypothesis we consider is considerably more restrictive than current approaches, which can be used much more generally. The null hypothesis for testing segregation or association can be described in two slightly different forms Dixon (1994):

(i) complete spatial randomness, that is, each class is distributed randomly throughout the area of interest. It describes both the arrangement of the locations and the association between classes.

(ii) random labeling of locations, which is less restrictive than spatial randomness, in the sense that arrangement of the locations can either be random or non-random.

Our conditional test is closer to the former in this regard. Pielou's test provide insight only on the association between classes, hence there is no assumption on the allocation of the observations, which makes it more appropriate for testing the null hypothesis of random labeling. Ripley's test can be used for both types of null hypotheses, in particular, it can be used to test a type of spatial randomness against another type of spatial randomness.

The test based on the mean domination number in Ceyhan and Priebe (2003b) is not a conditional test, but requires both $n$ and number of Delaunay triangles $J$ to be large. The comparison for a large but fixed $J$ is possible. Furthermore, under segregation alternatives, the Pitman asymptotic efficiency is not applicable to the mean domination number case, however, for large $n$ and $J$ we suggest the use of it over arc density since for each $\epsilon>0$, Hodges-Lehmann asymptotic efficiency is unbounded for the mean domination number case, while it is bounded for arc density case with $J>1$. As for the association alternative, HLAE suggests moderate $r$ values which has finite Hodges-Lehmann asymptotic efficiency. So again, for large $J$ and $n$ mean domination number is preferable. The basic advantage of $\rho_{n}(r)$ is that, it does not require $J$ to be large, so for small $J$ it is preferable.

Although the statistical analysis and the mathematical properties related to the $r$-factor proximity catch digraph are done in $\mathbb{R}^{2}$, the extension to $\mathbb{R}^{d}$ with $d>2$ is straightforward. See Ceyhan and Priebe Ceyhan and Priebe (2003b) for more detail on the construction of the associated proximity region in higher dimensions. Moreover, the geometry invariance, asymptotic normality of the $U$-statistic and consistency of the tests hold for $d>2$. 


\section{References}

Ceyhan, E. and Priebe, C. (2003a). Central similarity proximity maps in Delaunay tessellations. In Proceedings of the Joint Statistical Meeting, Statistical Computing Section, American Statistical Association.

Ceyhan, E. and Priebe, C. (2003b). The use of domination number of a random proximity catch digraph for testing segregation/association. Technical Report 642, Department of Applied Mathematics and Statistics, The Johns Hopkins University, Baltimore, MD, 21218. submitted for publication.

Coomes, D. A., Rees, M., and Turnbull, L. (1999). Identifying aggregation and association in fully mapped spatial data. Ecology, 80(2):554-565.

Cressie, N. A. C. (1991). Statistics for Spatial Data. Wiley, New York.

DeVinney, J., Priebe, C. E., Marchette, D. J., and Socolinsky, D. (2002). Random walks and catch digraphs in classification. http://www.galaxy.gmu.edu/interface/I02/I2002Proceedings/DeVinneyJason/DeVinneyJason.paper.pdf Proceedings of the $34^{\text {th }}$ Symposium on the Interface: Computing Science and Statistics, Vol. 34.

Dixon, P. M. (1994). Testing spatial segregation using a nearest-neighbor contingency table. Ecology, 75(7):1940-1948.

Eeden, C. V. (1963). The relation between Pitman's asymptotic relative efficiency of two tests and the correlation coefficient between their test statistics. The Annals of Mathematical Statistics, 34(4):14421451.

Gotelli, N. J. and Graves, G. R. (1996). Null Models in Ecology. Smithsonian Institution Press.

Hodges, J. L. J. and Lehmann, E. L. (1956). The efficiency of some nonparametric competitors of the $t$-test. The Annals of Mathematical Statistics, 27(2):324-335.

Janson, S., Łuczak, T., and Rucinński, A. (2000). Random Graphs. Wiley-Interscience Series in Discrete Mathematics and Optimization, John Wiley \& Sons, Inc., New York.

Jaromczyk, J. W. and Toussaint, G. T. (1992). Relative neighborhood graphs and their relatives. Proceedings of IEEE, 80:1502-1517.

Kendall, M. and Stuart, A. (1979). The Advanced Theory of Statistics, Volume 2., 4th edition. Griffin, London.

Lahiri, S. N. (1996). On consistency of estimators based on spatial data under infill asymptotics. Sankhya: The Indian Journal of Statistics, Series A, 58(3):403-417.

Lehmann, E. L. (1988). Nonparametrics: Statistical Methods Based on Ranks. Prentice-Hall, Upper Saddle River, NJ.

Marchette, D. J. and Priebe, C. E. (2003). Characterizing the scale dimension of a high dimensional classification problem. Pattern Recognition, 36(1):45-60.

Okabe, A., Boots, B., and Sugihara, K. (2000). Spatial Tessellations: Concepts and Applications of Voronoi Diagrams. Wiley.

Priebe, C. E., DeVinney, J. G., and Marchette, D. J. (2001). On the distribution of the domination number of random class catch cover digraphs. Statistics and Probability Letters, 55:239-246.

Priebe, C. E., Marchette, D. J., DeVinney, J., and Socolinsky, D. (2003a). Classification using class cover catch digraphs. Journal of Classification, 20(1):3-23.

Priebe, C. E., Solka, J. L., Marchette, D. J., and Clark, B. T. (2003b). Class cover catch digraphs for latent class discovery in gene expression monitoring by DNA microarrays. Computational Statistics and Data Analysis on Visualization, 43-4:621-632. 
Toussaint, G. T. (1980). The relative neighborhood graph of a finite planar set. Pattern Recognition, $12(4): 261-268$.

\section{Appendix 1: Derivation of $\mu(r)$ and $\nu(r)$}

In the standard equilateral triangle, let $\mathrm{y}_{1}=(0,0), \mathrm{y}_{2}=(1,0), \mathrm{y}_{3}=(1 / 2, \sqrt{3} / 2), M_{C}$ be the center of mass, $M_{j}$ be the midpoints of the edges $e_{j}$ for $j=1,2,3$. Then $M_{C}=(1 / 2, \sqrt{3} / 6), M_{1}=(3 / 4, \sqrt{3} / 4)$, $M_{2}=(1 / 4, \sqrt{3} / 4), M_{3}=(1 / 2,0)$.

Recall that $\mathbf{E}\left[\rho_{n}(r)\right]=\frac{1}{n(n-1)} \sum \sum_{i<j} \mathbf{E}\left[h_{i j}\right]=\frac{1}{2} \mathbf{E}\left[h_{12}\right]=\mu(r)=P\left(X_{j} \in N_{\mathcal{Y}}^{r}\left(X_{i}\right)\right)$.

Let $\mathcal{X}_{n}$ be a random sample of size $n$ from $\mathcal{U}(T(\mathcal{Y}))$. For $x_{1}=(u, v), \ell_{r}\left(x_{1}\right)=r v+r \sqrt{3} u-\sqrt{3} x$. Next, let $N_{1}:=\ell_{r}\left(x_{1}\right) \cap e_{3}$ and $N_{2}:=\ell_{r}\left(x_{1}\right) \cap e_{2}$. Then for $z_{1} \in T_{s}:=T\left(\mathrm{y}_{1}, M_{3}, M_{C}\right), N_{\mathcal{Y}}^{r}\left(z_{1}\right)=T\left(\mathrm{y}_{1}, N_{1}, N_{2}\right)$ provided that $\ell_{r}\left(x_{1}\right)$ is not outside of $T(\mathcal{Y})$, where

$$
N_{1}=\left(r\left(y_{1}+\sqrt{3} x_{1}\right) \sqrt{3} / 3,0\right) \text { and } N_{2}=\left(r\left(y_{1}+\sqrt{3} x_{1}\right) \sqrt{3} / 6,\left(y_{1}+\sqrt{3} x_{1}\right) r / 2\right) .
$$

Now we find $\mu(r)$ for $r \in[1, \infty)$.

First, observe that, by symmetry,

$$
\mu(r)=P\left(X_{2} \in N_{\mathcal{Y}}^{r}\left(X_{1}\right)\right)=6 P\left(X_{2} \in N_{\mathcal{Y}}^{r}\left(X_{1}\right), X_{1} \in T_{s}\right) .
$$

Let $\ell_{s}(r, x)$ be the line such that $r d\left(\mathrm{y}_{1}, \ell_{s}(r, x)\right)=d\left(\mathrm{y}_{1}, e_{1}\right)$ and $\ell_{s}(r, x) \cap T(\mathcal{Y}) \neq \emptyset$, so $\ell_{s}(r, x)=\sqrt{3}\left(\frac{1}{r}-x\right)$. Then if $x_{1} \in T_{s}$ is above $\ell_{s}(r, x)$ then $N_{\mathcal{Y}}^{r}\left(x_{1}\right)=T(\mathcal{Y})$, otherwise, $N_{\mathcal{Y}}^{r}\left(x_{1}\right)=T_{r}\left(x_{1}\right) \subsetneq T(\mathcal{Y})$.

For $r \in[1,3 / 2), \ell_{s}(r, x) \cap T_{s}=\emptyset$, so $N_{\mathcal{Y}}^{r}\left(x_{1}\right)=T_{r}\left(x_{1}\right) \subsetneq T(\mathcal{Y})$ for all $x \in T_{s}$. Then

$$
P\left(X_{2} \in N_{\mathcal{Y}}^{r}\left(X_{1}\right), X_{1} \in T_{s}\right)=\int_{0}^{1 / 2} \int_{0}^{x / \sqrt{3}} \frac{A\left(N_{\mathcal{Y}}^{r}\left(x_{1}\right)\right)}{A(T(\mathcal{Y}))^{2}} d y d x=\frac{37}{1296} r^{2} .
$$

where $A\left(N_{\mathcal{Y}}^{r}\left(x_{1}\right)\right)=\frac{\sqrt{3}}{12} r^{2}(y+\sqrt{3} x)^{2}$ and $A(T(\mathcal{Y}))=\sqrt{3} / 4$. Hence for $r \in[1,3 / 2), \mu(r)=\frac{37}{216} r^{2}$.

For $r \in[3 / 2,2), \ell_{s}(r, x)$ crosses through $\bar{M}_{3} M_{C}$. Let the $x$ coordinate of $\ell_{s}(r, x) \cap{\overline{y_{1}}}_{C}$ be $s_{1}$, then $s_{1}=3 /(4 r)$. See Figure 19 for the relative position of $\ell_{s}(r, x)$ and $T_{s}$.

Then

$$
\begin{aligned}
P & \left(X_{2} \in N_{\mathcal{Y}}^{r}\left(X_{1}\right), X_{1} \in T_{s}\right)=\int_{0}^{1 / 2} \int_{0}^{x / \sqrt{3}} \frac{A\left(N_{\mathcal{Y}}^{r}\left(x_{1}\right)\right)}{A(T(\mathcal{Y}))^{2}} d y d x \\
& =\int_{0}^{s_{1}} \int_{0}^{x / \sqrt{3}} \frac{A\left(N_{\mathcal{Y}}^{r}\left(x_{1}\right)\right)}{A(T(\mathcal{Y}))^{2}} d y d x+\int_{s_{1}}^{1 / 2} \int_{0}^{\ell_{s}(r, x)} \frac{A\left(N_{\mathcal{Y}}^{r}\left(x_{1}\right)\right)}{A(T(\mathcal{Y}))^{2}} d y d x+\int_{s_{1}}^{1 / 2} \int_{\ell_{s}(r, x)}^{x / \sqrt{3}} \frac{1}{A(T(\mathcal{Y}))} d y d x \\
& =-\frac{-36+r^{4}+64 r-32 r^{2}}{48 r^{2}} .
\end{aligned}
$$

Hence for $r \in[3 / 2,2), \mu(r)=-\frac{1}{8} r^{2}-8 r^{-1}+\frac{9}{2} r^{-2}+4$.

For $r \in[2, \infty), \ell_{s}(r, x)$ crosses through ${\overline{\mathrm{y}_{1} M_{3}}}_{3}$. Let the $x$ coordinate of $\ell_{s}(r, x) \cap{\overline{y_{1}} M_{3}}_{3}$ be $s_{2}$, then $s_{2}=1 / r$. See Figure 19

Then

$$
P\left(X_{2} \in N_{\mathcal{Y}}^{r}\left(X_{1}\right), X_{1} \in T_{s}\right)=\int_{0}^{s_{1}} \int_{0}^{x / \sqrt{3}} \frac{A\left(N_{\mathcal{Y}}^{r}\left(x_{1}\right)\right)}{A(T(\mathcal{Y}))^{2}} d y d x+\int_{s_{1}}^{s_{2}} \int_{0}^{\ell_{s}(r, x)} \frac{A\left(N_{\mathcal{Y}}^{r}\left(x_{1}\right)\right)}{A(T(\mathcal{Y}))^{2}} d y d x
$$

$$
D R A-1+\int_{s_{1}}^{s_{2}} \int_{\ell_{s}(r, x)}^{x / \sqrt{3}} \frac{1}{A(T(\mathcal{Y}))} d y d x+\int_{s_{2}}^{1 / 2} \int_{0}^{x / \sqrt{3}} \frac{1}{A(T(\mathcal{Y}))} d y d x=\frac{1}{12} \frac{-3+2 r^{2}}{r^{2}} \text {. }
$$




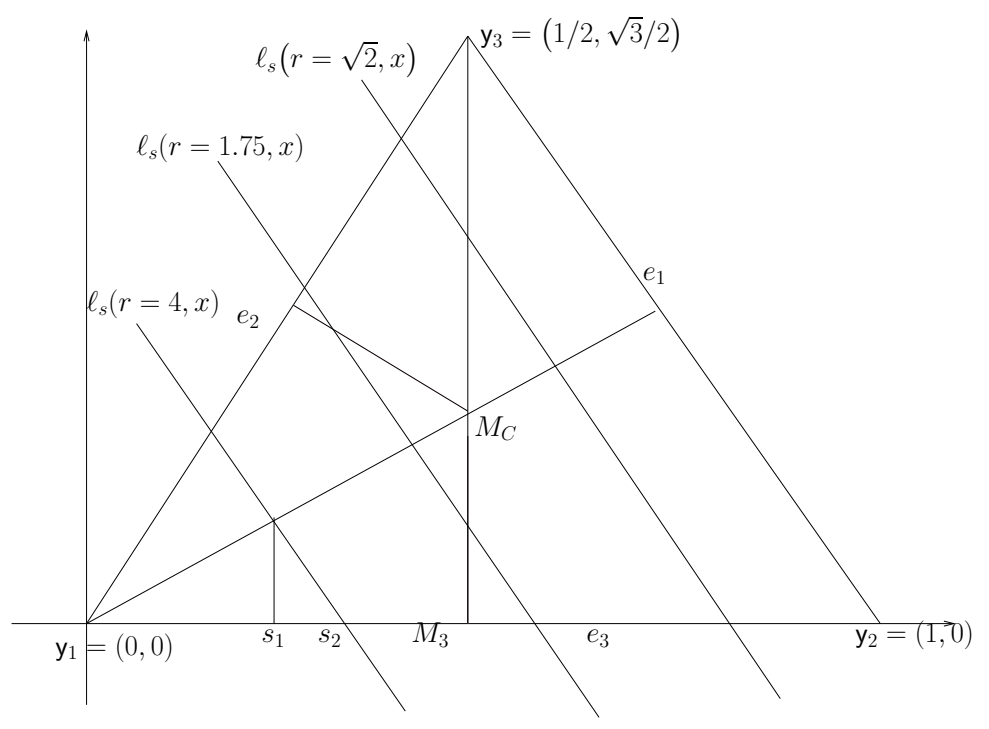

Figure 19: The cases for relative position of $\ell_{s}(r, x)$ with various $r$ values.

Hence for $r \in[2, \infty), \mu(r)=1-\frac{3}{2} r^{-2}$.

For $r=\infty, \mu(r)=1$ follows trivially.

To find $\mathbf{C o v}\left[h_{12}, h_{13}\right]$, we introduce a related concept.

Definition: Let $(\Omega, \mathcal{M})$ be a measurable space and consider the proximity map $N: \Omega \times \wp(\Omega) \rightarrow \wp(\Omega)$, where $\wp(\cdot)$ represents the power set functional. For $B \subset \Omega$, the $\Gamma_{1}$-region, $\Gamma_{1}(\cdot)=\Gamma_{1}(\cdot, N): \Omega \rightarrow \wp(\Omega)$ associates the region $\Gamma_{1}(B):=\{z \in \Omega: B \subseteq N(z)\}$ with each set $B \subset \Omega$. For $x \in \Omega$, we denote $\Gamma_{1}(\{x\})$ as $\Gamma_{1}(x)$. Note that $\Gamma_{1}$-region depends on proximity region $N(\cdot)$.

Furthermore, let $\Gamma_{1}\left(\cdot, N_{\mathcal{Y}}^{r}\right)$ be the $\Gamma_{1}$-region associated with $N_{\mathcal{Y}}^{r}(\cdot)$, let $A_{i j}$ be the event that $\left\{X_{i} X_{j} \in\right.$ $\mathcal{A}\}=\left\{X_{i} \in N_{\mathcal{Y}}^{r}\left(X_{j}\right)\right\}$, then $h_{i j}=I\left(A_{i j}\right)+I\left(A_{j i}\right)$. Let

$P_{2 N}^{r}:=P\left(\left\{X_{2}, X_{3}\right\} \subset N_{\mathcal{Y}}^{r}\left(X_{1}\right)\right), \quad P_{M}^{r}:=P\left(X_{2} \in N_{\mathcal{Y}}^{r}\left(X_{1}\right), X_{3} \in \Gamma_{1}\left(X_{1}, N_{\mathcal{Y}}^{r}\right), \quad P_{2 G}^{r}:=P\left(\left\{X_{2}, X_{3}\right\} \subset \Gamma_{1}\left(X_{1}, N_{\mathcal{Y}}^{r}\right)\right)\right.$.

Then $\mathbf{C o v}\left[h_{12}, h_{13}\right]=\mathbf{E}\left[h_{12} h_{13}\right]-\mathbf{E}\left[h_{12}\right] \mathbf{E}\left[h_{13}\right]$ where

$\mathbf{E}\left[h_{12} h_{13}\right]=\mathbf{E}\left[\left(\mathbf{I}\left(A_{12}\right)+\mathbf{I}\left(A_{21}\right)\right)\left(\mathbf{I}\left(A_{13}\right)+\mathbf{I}\left(A_{31}\right)\right]\right.$

$=P\left(A_{12} \cap A_{13}\right)+P\left(A_{12} \cap A_{31}\right)+P\left(A_{21} \cap A_{13}\right)+P\left(A_{21} \cap A_{31}\right)$.

$=P\left(\left\{X_{2}, X_{3}\right\} \subset N_{\mathcal{Y}}^{r}\left(X_{1}\right)\right)+2 P\left(X_{2} \in N_{\mathcal{Y}}^{r}\left(X_{1}\right), X_{3} \in \Gamma_{1}\left(X_{1}, N_{\mathcal{Y}}^{r}\right)\right)+P\left(\left\{X_{2}, X_{3}\right\} \subset \Gamma_{1}\left(X_{1}, N_{\mathcal{Y}}^{r}\right)\right)$

$=P_{2 N}^{r}+2 P_{M}^{r}+P_{2 G}^{r}$.

So $\nu(r)=\operatorname{Cov}\left[h_{12}, h_{13}\right]=\left(P_{2 N}^{r}+2 P_{M}^{r}+P_{2 G}^{r}\right)-[2 \mu(r)]^{2}$.

Furthermore, for any $x_{1}=(u, v) \in T(\mathcal{Y}), \Gamma_{1}\left(x_{1}, N_{\mathcal{Y}}^{r}\right)$ is a convex or nonconvex polygon. Let $\xi_{j}(r, x)$ be the line between $x_{1}$ and the vertex $\mathrm{y}_{j}$ parallel to the edge $e_{j}$ such that $r d\left(\mathrm{y}_{j}, \xi_{j}(r, x)\right)=d\left(\mathrm{y}_{j}, \ell_{r}\left(x_{1}\right)\right)$ for $j=$ $1,2,3$. Then $\Gamma_{1}\left(x_{1}, N_{\mathcal{Y}}^{r}\right) \cap R\left(\mathrm{y}_{j}\right)$ is bounded by $\xi_{j}(r, x)$ and the median lines.

For $x_{1}=(u, v), \xi_{1}(r, x)=-\sqrt{3} x+(v+\sqrt{3} u) / r, \xi_{2}(r, x)=(v+\sqrt{3} r(x-1)+\sqrt{3}(1-u)) / r$ and $\xi_{3}(r, x)=$ $(\sqrt{3}(r-1)+2 v) /(2 r)$.

To find the covariance, we need to find the possible types of $\Gamma_{1}\left(x_{1}, N_{\mathcal{Y}}^{r}\right)$ and $N_{\mathcal{Y}}^{r}\left(x_{1}\right)$ for $r \in[1, \infty)$. First we find the possible intersection points of $\ell_{V}(x)$ with $\partial(T(\mathcal{Y}))$ and $\partial\left(R\left(\mathrm{y}_{j}\right)\right)$ for $j=1,2,3$. Let

$G_{1}=\xi_{1}(r, x) \cap e_{3}, G_{2}=\xi_{2}(r, x) \cap e_{3},-G_{3}=\xi_{2}(r, x) \cap e_{1}, G_{4}=\xi_{3}(r, x) \cap e_{1}, G_{5}=\xi_{3}(r, x) \cap e_{2},-G_{6}=\xi_{1}(r, x) \cap e_{2}$. 


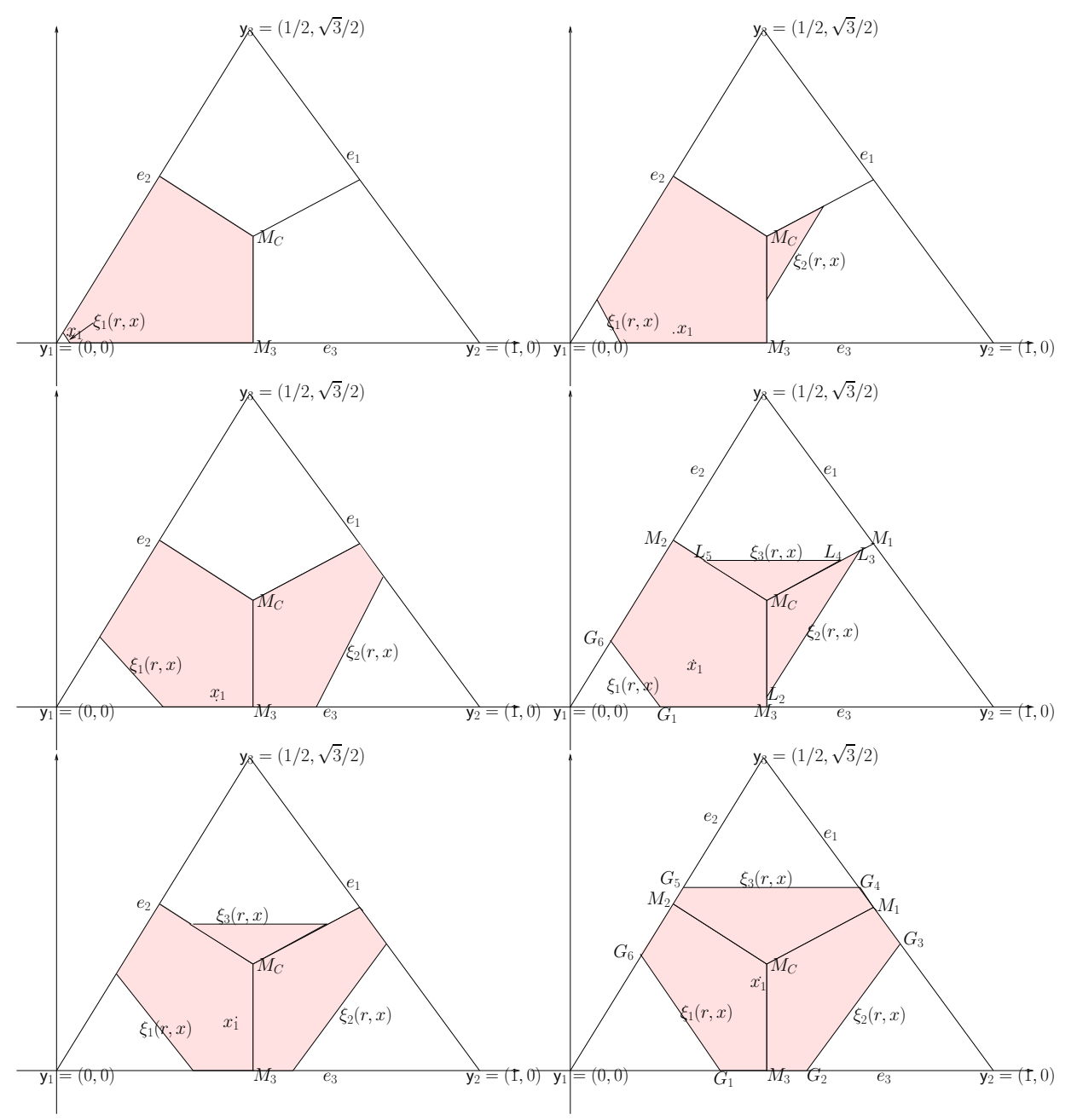

Figure 20: The prototypes of the six cases for $\Gamma_{1}\left(x_{1}, N_{\mathcal{Y}}^{r}\right)$ for $x \in T\left(\mathrm{y}_{1}, M_{3}, M_{C C}\right)$ for $r \in[4 / 3,3 / 2)$.

Then, for example, $G_{5}=\left(\frac{(\sqrt{3} r-\sqrt{3}+2 y) \sqrt{3}}{6 r}, \frac{\sqrt{3} r-\sqrt{3}+2 y}{2 r}\right)$. Furthermore, let $L_{1}=\xi_{1}(r, x) \cap \bar{M}_{1} M_{C}, \quad L_{2}=\xi_{2}(r, x) \cap{\overline{M_{1} M_{C}}}_{C}, \quad L_{3}=\xi_{2}(r, x) \cap \bar{M}_{2} M_{C}, \quad L_{4}=\xi_{3}(r, x) \cap \bar{M}_{2} M_{C}, \quad L_{5}=$ $\xi_{3}(r, x) \cap \bar{M}_{3} M_{C}, \quad L_{6}=\xi_{1}(r, x) \cap \bar{M}_{3} M_{C}$.

Then for example $L_{5}=\left(-\frac{(\sqrt{3} r-3 \sqrt{3}+6 y) \sqrt{3}}{6 r}, \frac{\sqrt{3} r-\sqrt{3}+2 y}{2 r}\right)$. Then $\Gamma_{1}\left(x_{1}, N_{\mathcal{Y}}^{r}\right)$ is a polygon whose vertices are a subset of the $\mathrm{y}_{j}, M_{C}, M_{j}, j=1,2,3$ and $G_{j}, L_{j}, j=1, \ldots, 6$.

See Figure 20 for the prototypes of $\Gamma_{1}\left(x_{1}, N_{\mathcal{Y}}^{r}\right)$ with $r \in[4 / 3,3 / 2)$.

We partition $[1, \infty)$ with respect to the types of $N_{\mathcal{Y}}^{r}\left(x_{1}\right)$ and $\Gamma_{1}\left(x_{1}, N_{\mathcal{Y}}^{r}\right)$ into $[1,4 / 3),[4 / 3,3 / 2),[3 / 2,2),[2, \infty)$. For demonstrative purposes we pick the interval $[4 / 3,3 / 2)$. For $r \in\left[\frac{4}{3}, \frac{3}{2}\right)$, there are six cases regarding $\Gamma_{1}\left(x_{1}, N_{\mathcal{Y}}^{r}\right)$ and one case for $N_{\mathcal{Y}}^{r}\left(x_{1}\right)$. Each case $j$ corresponds to the region $R_{j}$ in Figure 21 where $s_{1}=1-2 r / 3, s_{2}=3 / 2-r, s_{3}=1-r / 2, s_{4}=3 / 2-5 r / 6, s_{5}=3 / 2-3 r / 4$.

Let $\mathscr{P}\left(a_{1}, a_{2}, \ldots, a_{n}\right)$ denote the polygon with vertices $a_{1}, a_{2}, \ldots, a_{n}$, then, for $x_{1}=(x, y) \in R_{j}, j=$ $1, \ldots, 6, \Gamma_{1}\left(x_{1}, N_{\mathcal{Y}}^{r}\right)$ are $\mathscr{P}\left(G_{1}, M_{1}, M_{C}, M_{3}, G_{6}\right), \mathscr{P}\left(G_{1}, M_{1}, L_{2}, L_{3}, M_{C}, M_{3}, G_{6}\right), \mathscr{P}\left(G_{1}, G_{2}, G_{3}, M_{2}, M_{C}, M_{3}, G_{6}\right)$, $\mathscr{P}\left(G_{1}, M_{1}, L_{2}, L_{3}, L_{4}, L_{5}, M_{3}, G_{6}\right), \mathscr{P}\left(G_{1}, G_{2}, G_{3}, M_{2}, L_{4}, L_{5}, M_{3}, G_{6}\right)$ and $\mathscr{P}\left(G_{1}, G_{2}, G_{3}, G_{4}, G_{5}, G_{6}\right)$, respectively.

The explicit forms of $R_{j}, j=1, \ldots, 6$ are as follows: 


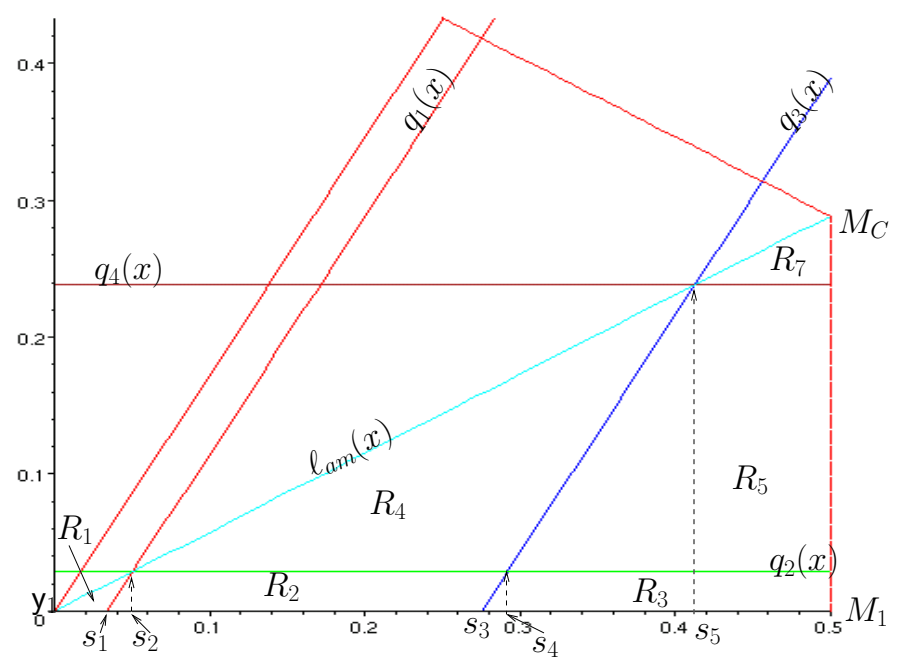

Figure 21: The regions corresponding to the six cases for $r \in[4 / 3,3 / 2)$

$$
\begin{aligned}
& R_{1}=\left\{(x, y) \in\left[0, s_{1}\right] \times\left[0, \ell_{a m}(x)\right] \cup\left[s_{1}, s_{2}\right] \times\left[q_{1}(x), \ell_{a m}(x)\right]\right\} \\
& R_{2}=\left\{(x, y) \in\left[s_{1}, s_{2}\right] \times\left[0, q_{1}(x)\right] \cup\left[s_{2}, s_{3}\right] \times\left[0, q_{2}(x)\right] \cup\left[s_{3}, s_{4}\right] \times\left[q_{3}(x), q_{2}(x)\right]\right\} \\
& R_{3}=\left\{(x, y) \in\left[s_{3}, s_{4}\right] \times\left[0, q_{3}(x)\right] \cup\left[s_{4}, 1 / 2\right] \times\left[0, q_{2}(x)\right]\right\} \\
& R_{4}=\left\{(x, y) \in\left[s_{2}, s_{4}\right] \times\left[q_{2}(x), \ell_{a m}(x)\right] \cup\left[s_{4}, s_{6}\right] \times\left[q_{3}(x), \ell_{a m}(x)\right]\right\} \\
& R_{5}=\left\{(x, y) \in\left[s_{4}, s_{6}\right] \times\left[q_{2}(x), q_{3}(x)\right] \cup\left[s_{6}, 1 / 2\right] \times\left[q_{2}(x), q_{4}(x)\right]\right\} \\
& R_{6}=\left\{(x, y) \in\left[s_{6}, 1 / 2\right] \times\left[q_{4}(x), \ell_{a m}(x)\right]\right\}
\end{aligned}
$$

where $\ell_{a m}(x)=x / \sqrt{3}, q_{1}(x)=(2 r-3) / \sqrt{3}+\sqrt{3} x, q_{2}(x)=\sqrt{3}(1 / 2-r / 3), q_{3}(x)=\sqrt{3}(x-1+r / 2)$, and $q_{4}(x)=\sqrt{3}(1 / 2-r / 4)$.

Then $P\left(\left\{X_{2}, X_{3}\right\} \subset N_{\mathcal{Y}}^{r}\left(X_{1}\right)\right)=\frac{781}{19440} r^{4}$. (We use the same limits of integration in $\mu(r)$ calculations with the integrand being $A\left(N_{\mathcal{Y}}^{r}\left(x_{1}\right)\right)^{2} / A(T(\mathcal{Y}))^{3}$. Then

Next, by symmetry, $P\left(\left\{X_{2}, X_{3}\right\} \subset \Gamma_{1}\left(X_{1}, N_{\mathcal{Y}}^{r}\right)\right)=6 P\left(\left\{X_{2}, X_{3}\right\} \subset \Gamma_{1}\left(X_{1}, N_{\mathcal{Y}}^{r}\right), X_{1} \in T\left(\mathrm{y}, M_{3}, M_{C}\right)\right)$.

$$
P\left(\left\{X_{2}, X_{3}\right\} \subset \Gamma_{1}\left(X_{1}, N_{\mathcal{Y}}^{r}\right), X_{1} \in T\left(\mathrm{y}, M_{3}, M_{C}\right)\right)=\sum_{j=1}^{6} P\left(\left\{X_{2}, X_{3}\right\} \subset \Gamma_{1}\left(X_{1}, N_{\mathcal{Y}}^{r}\right), X_{1} \in R_{j}\right) .
$$

For example, for $x_{1} \in R_{4}$,

$$
\begin{aligned}
P\left(\left\{X_{2}, X_{3}\right\} \subset \Gamma_{1}\left(X_{1}, N_{\mathcal{Y}}^{r}\right), X_{1} \in R_{4}\right)=\int_{s_{2}}^{s_{4}} \int_{q_{2}(x)}^{\ell_{a m}(x)} \frac{A\left(\Gamma_{1}\left(x_{1}, N_{\mathcal{Y}}^{r}\right)\right)^{2}}{A(T(\mathcal{Y}))^{3}} d y d x \\
\quad+\int_{s_{4}}^{s_{6}} \int_{q_{3}(x)}^{\ell_{a m}(x)} \frac{A\left(\Gamma_{1}\left(x_{1}, N_{\mathcal{Y}}^{r}\right)\right)^{2}}{A(T(\mathcal{Y}))^{3}} d y d x=\frac{9637 r^{4}-89640 r^{3}+288360 r^{2}-362880 r+155520}{349920 r^{2}} .
\end{aligned}
$$

where $A\left(\Gamma_{1}\left(x_{1}, N_{\mathcal{Y}}^{r}\right)\right)=\frac{\sqrt{3}\left(9 r^{2}+18-24 r+4 \sqrt{3} r y-18 x+6 x^{2}+14 y^{2}+12 r x-8 x \sqrt{3} y-6 \sqrt{3} y\right)}{12 r^{2}}$.

Similarly, we calculate for $j=1,2,3,5,6$ and get

$$
\begin{aligned}
P\left(\left\{X_{2}, X_{3}\right\} \subset \Gamma_{1}\left(X_{1}, N_{\mathcal{Y}}^{r}\right)\right) & =6\left(\frac{-47880 r^{5}-38880 r^{2}+25687 r^{6}-1080 r^{4}+60480 r^{3}+3888}{349920 r^{4}}\right) \\
-- & =\frac{-47880 r^{5}-38880 r^{2}+25687 r^{6}-1080 r^{4}+60480 r^{3}+3888}{58320 r^{4}} .
\end{aligned}
$$


Furthermore, $P\left(X_{2} \in N_{\mathcal{Y}}^{r}\left(X_{1}\right), X_{3} \in \Gamma_{1}\left(X_{1}, N_{\mathcal{Y}}^{r}\right), X_{1} \in T\left(\mathrm{y}, M_{3}, M_{C}\right)\right)=\sum_{j=1}^{6} P\left(X_{2} \in N_{\mathcal{Y}}^{r}\left(X_{1}\right), X_{3} \in\right.$ $\left.\Gamma_{1}\left(X_{1}, N_{\mathcal{Y}}^{r}\right), X_{1} \in R_{j}\right)$.

For example, $x_{1} \in R_{4}$, we get $-\frac{1}{466560} r^{2}\left(207360+404640 r^{2}-483840 r-142920 r^{3}+17687 r^{4}\right)$ by using the same integration limits as above, with the integrand being $A\left(N_{\mathcal{Y}}^{r}\left(x_{1}\right)\right) A\left(\Gamma_{1}\left(x_{1}, N_{\mathcal{Y}}^{r}\right)\right) / A(T(\mathcal{Y}))^{3}$.

Similarly, we calculate for $j=1,2,3,5,6$ and get

$$
\begin{aligned}
P\left(X_{2} \in N_{\mathcal{Y}}^{r}\left(X_{1}\right), X_{3} \in \Gamma_{1}\left(X_{1}, N_{\mathcal{Y}}^{r}\right)\right) & =6\left(\frac{5467}{2799360} r^{6}-\frac{35}{2592} r^{5}+\frac{37}{1296} r^{4}-\frac{13}{648} r^{2}+\frac{83}{12960}\right) \\
& =\frac{5467}{466560} r^{6}-\frac{35}{432} r^{5}+\frac{37}{216} r^{4}-\frac{13}{108} r^{2}+\frac{83}{2160} .
\end{aligned}
$$

So, $\mathbf{E}\left[h_{12} h_{13}\right]=\left[5467 r^{10}-37800 r^{9}+89292 r^{8}+46588 r^{6}-191520 r^{5}+13608 r^{4}+241920 r^{3}-155520 r^{2}+\right.$ $15552] /\left[233280 r^{4}\right]$.

Thus, for $r \in[4 / 3,3 / 2), \nu(r)=\left[5467 r^{10}-37800 r^{9}+61912 r^{8}+46588 r^{6}-191520 r^{5}+13608 r^{4}+241920 r^{3}-\right.$ $\left.155520 r^{2}+15552\right] /\left[233280 r^{4}\right]$.

\section{Appendix 2: $\mu(r, \epsilon)$ for Segregation and Association Alternatives}

Derivation of $\mu(r, \epsilon)$ involves detailed geometric calculations and partitioning of the space of $\left(r, \epsilon, x_{1}\right)$ for $r \in[1, \infty), \epsilon \in[0, \sqrt{3} / 3)$, and $x_{1} \in T_{s}$.

\section{$\mu_{S}(r, \epsilon)$ Under Segregation Alternatives}

Under segregation, we compute $\mu_{S}(r, \epsilon)$ explicitly. For $\epsilon \in[0, \sqrt{3} / 8), \mu_{S}(r, \epsilon)=\sum_{j=1}^{7} \mu_{1, j}(r, \epsilon) \mathbf{I}\left(r \in \mathcal{I}_{j}\right)$ where

$$
\begin{aligned}
\mu_{1,1}(r, \epsilon) & =-\frac{576 r^{2} \epsilon^{4}-1152 \epsilon^{4}-37 r^{2}+288 \epsilon^{2}}{216(2 \epsilon+1)^{2}(2 \epsilon-1)^{2}}, \\
\mu_{1,2}(r, \epsilon) & =-\left[576 r^{4} \epsilon^{4}-1152 r^{2} \epsilon^{4}+91 r^{4}+512 \sqrt{3} r^{3} \epsilon+2592 r^{2} \epsilon^{2}+1536 \sqrt{3} r \epsilon^{3}+1152 \epsilon^{4}\right. \\
& -768 r^{3}-2304 \sqrt{3} r^{2} \epsilon-6912 r \epsilon^{2}-2304 \sqrt{3} \epsilon^{3}+1728 r^{2}+3456 \sqrt{3} r \epsilon+5184 \epsilon^{2} \\
& -1728 r-1728 \sqrt{3} \epsilon+648] /\left[216 r^{2}(2 \epsilon+1)^{2}(2 \epsilon-1)^{2}\right], \\
\mu_{1,3}(r, \epsilon) & =-\left[192 r^{4} \epsilon^{4}-384 r^{2} \epsilon^{4}+9 r^{4}+864 r^{2} \epsilon^{2}+512 \sqrt{3} r \epsilon^{3}+384 \epsilon^{4}-2304 r \epsilon^{2}-768 \sqrt{3} \epsilon^{3}\right. \\
& \left.-288 r^{2}+1728 \epsilon^{2}+576 r-324\right] /\left[72 r^{2}(2 \epsilon+1)^{2}(2 \epsilon-1)^{2}\right], \\
\mu_{1,4}(r, \epsilon) & =-\left[192 r^{4} \epsilon^{4}-384 r^{2} \epsilon^{4}-9 r^{4}-96 \sqrt{3} r^{3} \epsilon+288 r^{2} \epsilon^{2}-128 \epsilon^{4}+144 r^{3}+576 \sqrt{3} r^{2} \epsilon+256\right. \\
& \left.\sqrt{3} \epsilon^{3}-720 r^{2}-1152 \sqrt{3} r \epsilon-576 \epsilon^{2}+1152 r+768 \sqrt{3} \epsilon-612\right] /\left[72 r^{2}(2 \epsilon+1)^{2}(2 \epsilon-1)^{2}\right], \\
\mu_{1,5}(r, \epsilon) & =-\frac{48 r^{4} \epsilon^{4}-96 r^{2} \epsilon^{4}+72 r^{2} \epsilon^{2}-32 \epsilon^{4}+64 \sqrt{3} \epsilon^{3}-18 r^{2}-144 \epsilon^{2}+27}{18 r^{2}(2 \epsilon+1)^{2}(2 \epsilon-1)^{2}}, \\
\mu_{1,6}(r, \epsilon) & =\frac{48 r^{4} \epsilon^{4}+256 r^{3} \epsilon^{4}-128 \sqrt{3} r^{3} \epsilon^{3}+288 r^{2} \epsilon^{4}-192 \sqrt{3} r^{2} \epsilon^{3}+72 r^{2} \epsilon^{2}+18 r^{2}+48 \sqrt{3} \epsilon-45}{18(2 \epsilon+1)^{2}(2 \epsilon-1)^{2} r^{2}}, \\
\mu_{1,7}(r, \epsilon) & =1,
\end{aligned}
$$

with the corresponding intervals $\mathcal{I}_{1}=[1,3 / 2-\sqrt{3} \epsilon), \mathcal{I}_{2}=[3 / 2-\sqrt{3} \epsilon, 3 / 2), \mathcal{I}_{3}=[3 / 2,2-4 \epsilon / \sqrt{3})$, $\mathcal{I}_{4}=[2-4 \epsilon / \sqrt{3}, 2), \mathcal{I}_{5}=[2, \sqrt{3} /(2 \epsilon)-1), \mathcal{I}_{6}=[\sqrt{3} /(2 \epsilon)-1, \sqrt{3} /(2 \epsilon))$, and $\mathcal{I}_{7}=[\sqrt{3} /(2 \epsilon), \infty)$.

For $\epsilon \in[\sqrt{3} / 8, \sqrt{3} / 6), \mu_{S}(r, \epsilon)=\sum_{j=1}^{7} \mu_{2, j}(r, \epsilon) \mathbf{I}\left(r \in \mathcal{I}_{j}\right)$ where $\mu_{2, j}(r, \epsilon)=\mu_{1, j}(r, \epsilon)$ for $j=1,2,4,5,6$, 
and for $j=3,7$,

$$
\begin{aligned}
\mu_{2,3}(r, \epsilon) & =-\left[576 r^{4} \epsilon^{4}-1152 r^{2} \epsilon^{4}+37 r^{4}+224 \sqrt{3} r^{3} \epsilon+864 r^{2} \epsilon^{2}-384 \epsilon^{4}-336 r^{3}-576 \sqrt{3} r^{2} \epsilon\right. \\
& \left.+768 \sqrt{3} \epsilon^{3}+432 r^{2}-1728 \epsilon^{2}+576 \sqrt{3} \epsilon-216\right] /\left[216 r^{2}(2 \epsilon+1)^{2}(2 \epsilon-1)^{2}\right], \\
\mu_{2,7}(r, \epsilon) & =1,
\end{aligned}
$$

with the corresponding intervals $\mathcal{I}_{1}=[1,3 / 2-\sqrt{3} \epsilon), \mathcal{I}_{2}=[3 / 2-\sqrt{3} \epsilon, 2-4 \epsilon / \sqrt{3}), \mathcal{I}_{3}=[2-4 \epsilon / \sqrt{3}, 3 / 2)$, $\mathcal{I}_{4}=[3 / 2,2), \mathcal{I}_{5}=[2, \sqrt{3} /(2 \epsilon)-1), \mathcal{I}_{6}=[\sqrt{3} /(2 \epsilon)-1, \sqrt{3} /(2 \epsilon))$, and $\mathcal{I}_{5}=[\sqrt{3} /(2 \epsilon), \infty)$.

For $\epsilon \in[\sqrt{3} / 6, \sqrt{3} / 4), \mu_{S}(r, \epsilon)=\sum_{j=1}^{6} \mu_{3, j}(r, \epsilon) \mathbf{I}\left(r \in \mathcal{I}_{j}\right)$ where $\mu_{3,1}(r, \epsilon)=\mu_{1,2}(r, \epsilon)$ and

$$
\begin{aligned}
\mu_{3,2}(r, \epsilon) & =-\left[576 r^{4} \epsilon^{4}-1152 r^{2} \epsilon^{4}+37 r^{4}+224 \sqrt{3} r^{3} \epsilon+864 r^{2} \epsilon^{2}-384 \epsilon^{4}-336 r^{3}-576 \sqrt{3} r^{2} \epsilon\right. \\
& \left.+768 \sqrt{3} \epsilon^{3}+432 r^{2}-1728 \epsilon^{2}+576 \sqrt{3} \epsilon-216\right] /\left[216 r^{2}(2 \epsilon+1)^{2}(2 \epsilon-1)^{2}\right], \\
\mu_{3,3}(r, \epsilon) & =\left[576 r^{2} \epsilon^{4}+3072 r \epsilon^{4}-1536 \sqrt{3} r \epsilon^{3}+3456 \epsilon^{4}-2304 \sqrt{3} \epsilon^{3}-37 r^{2}-224 \sqrt{3} r \epsilon\right. \\
& \left.+864 \epsilon^{2}+336 r+576 \sqrt{3} \epsilon-432\right] /\left[216(2 \epsilon+1)^{2}(2 \epsilon-1)^{2}\right], \\
\mu_{3,4}(r, \epsilon) & =\left[192 r^{4} \epsilon^{4}+1024 r^{3} \epsilon^{4}-512 \sqrt{3} r^{3} \epsilon^{3}+1152 r^{2} \epsilon^{4}-768 \sqrt{3} r^{2} \epsilon^{3}+9 r^{4}+96 \sqrt{3} r^{3} \epsilon+288 r^{2} \epsilon^{2}\right. \\
& \left.-144 r^{3}-576 \sqrt{3} r^{2} \epsilon+720 r^{2}+1152 \sqrt{3} r \epsilon-1152 r-576 \sqrt{3} \epsilon+540\right] /\left[72 r^{2}(2 \epsilon+1)^{2}(2 \epsilon-1)^{2}\right], \\
\mu_{3,5}(r, \epsilon) & =\frac{48 r^{4} \epsilon^{4}+256 r^{3} \epsilon^{4}-128 \sqrt{3} r^{3} \epsilon^{3}+288 r^{2} \epsilon^{4}-192 \sqrt{3} r^{2} \epsilon^{3}+72 r^{2} \epsilon^{2}+18 r^{2}+48 \sqrt{3} \epsilon-45}{18 r^{2}(2 \epsilon+1)^{2}(2 \epsilon-1)^{2}}, \\
\mu_{3,6}(r, \epsilon) & =1,
\end{aligned}
$$

with the corresponding intervals $\mathcal{I}_{1}=[1,2-4 \epsilon / \sqrt{3}), \mathcal{I}_{2}=[2-4 \epsilon / \sqrt{3}, \sqrt{3} /(2 \epsilon)-1), \mathcal{I}_{3}=[\sqrt{3} /(2 \epsilon)-1,3 / 2)$, $\mathcal{I}_{4}=[3 / 2,2), \mathcal{I}_{5}=[2, \sqrt{3} /(2 \epsilon))$, and $\mathcal{I}_{5}=[\sqrt{3} /(2 \epsilon), \infty)$.

For $\epsilon \in[\sqrt{3} / 4, \sqrt{3} / 3), \mu_{S}(r, \epsilon)=\sum_{j=1}^{3} \mu_{4, j}(r, \epsilon) \mathbf{I}\left(r \in \mathcal{I}_{j}\right)$ where

$$
\begin{aligned}
\mu_{4,1}(r, \epsilon) & =-\frac{9 r^{2} \epsilon^{2}+2 \sqrt{3} r^{2} \epsilon+48 r \epsilon^{2}+r^{2}-16 \sqrt{3} r \epsilon-90 \epsilon^{2}-12 r+36 \sqrt{3} \epsilon}{18(3 \epsilon-\sqrt{3})^{2}}, \\
\mu_{4,2}(r, \epsilon) & =-\left[9 r^{4} \epsilon^{4}-4 \sqrt{3} r^{4} \epsilon^{3}+48 r^{3} \epsilon^{4}-48 \sqrt{3} r^{3} \epsilon^{3}-90 r^{2} \epsilon^{4}+36 r^{3} \epsilon^{2}+96 \sqrt{3} r^{2} \epsilon^{3}-126 r^{2} \epsilon^{2}\right. \\
& -32 \sqrt{3} r \epsilon^{3}-48 \epsilon^{4}+36 \sqrt{3} r^{2} \epsilon+144 r \epsilon^{2}+96 \sqrt{3} \epsilon^{3}-18 r^{2}-72 \sqrt{3} r \epsilon-216 \epsilon^{2}+36 r \\
& +72 \sqrt{3} \epsilon-27] /\left[2(3 \epsilon-\sqrt{3})^{4} r^{2}\right], \\
\mu_{4,3}(r, \epsilon) & =1,
\end{aligned}
$$

with the corresponding intervals $\mathcal{I}_{1}=[1,3-2 \epsilon / \sqrt{3}), \mathcal{I}_{2}=[3-2 \epsilon / \sqrt{3}, \sqrt{3} / \epsilon-2)$, and $\mathcal{I}_{3}=[\sqrt{3} / \epsilon-2, \infty)$. 


\section{$\mu_{A}(r, \epsilon)$ Under Association Alternatives}

Under association, we compute $\mu_{A}(r, \epsilon)$ explicitly. For $\epsilon \in[0,(7 \sqrt{3}-3 \sqrt{15}) / 12 \approx .042), \mu_{A}(r, \epsilon)=$ $\sum_{j=1}^{6} \mu_{1, j}(r, \epsilon) \mathbf{I}\left(r \in \mathcal{I}_{j}\right)$ where

$$
\begin{aligned}
\mu_{1,1}(r, \epsilon) & =-\left[3456 \epsilon^{4} r^{4}+9216 \epsilon^{4} r^{3}-3072 \sqrt{3} \epsilon^{3} r^{4}-17280 \epsilon^{4} r^{2}-3072 \sqrt{3} \epsilon^{3} r^{3}+2304 \epsilon^{2} r^{4}\right. \\
& +4608 \sqrt{3} \epsilon^{3} r^{2}-2304 \epsilon^{2} r^{3}+6336 \epsilon^{4}+6144 \sqrt{3} \epsilon^{3} r+6912 \epsilon^{2} r^{2}+512 \sqrt{3} \epsilon r^{3} \\
& -101 r^{4}-6144 \sqrt{3} \epsilon^{3}-11520 \epsilon^{2} r-1536 \sqrt{3} \epsilon r^{2}+256 r^{3}+5760 \epsilon^{2}+1536 \sqrt{3} \epsilon r \\
& \left.-384 r^{2}-512 \sqrt{3} \epsilon+256 r-64\right] /\left[24(6 \epsilon+\sqrt{3})^{2}(6 \epsilon-\sqrt{3})^{2} r^{2}\right], \\
\mu_{1,2}(r, \epsilon) & =-\left[1728 \epsilon^{4} r^{4}-1536 \sqrt{3} \epsilon^{3} r^{4}-31104 \epsilon^{4} r^{2}+1152 \epsilon^{2} r^{4}+15552 \epsilon^{4}+10368 \epsilon^{2} r^{2}-37 r^{4}\right. \\
& \left.-20736 \epsilon^{2} r+10368 \epsilon^{2}\right] /\left[24(6 \epsilon+\sqrt{3})^{2}(6 \epsilon-\sqrt{3})^{2} r^{2}\right], \\
\mu_{1,3}(r, \epsilon) & =\left[-2592 \epsilon^{4} r^{4}-2304 \sqrt{3} \epsilon^{3} r^{4}-46656 \epsilon^{4} r^{2}+1728 \epsilon^{2} r^{4}+10656 \epsilon^{4}-9216 \sqrt{3} \epsilon^{3} r\right. \\
& +9072 \epsilon^{2} r^{2}-432 \sqrt{3} \epsilon r^{3}-15 r^{4}+12288 \sqrt{3} \epsilon^{3}-13824 \epsilon^{2} r+1728 \sqrt{3} \epsilon r^{2}-216 r^{3} \\
& \left.+4032 \epsilon^{2}-2304 \sqrt{3} \epsilon r+432 r^{2}+1024 \sqrt{3} \epsilon-384 r+128\right] /\left[36(6 \epsilon+\sqrt{3})^{2}(6 \epsilon-\sqrt{3})^{2} r^{2}\right], \\
\mu_{1,4}(r, \epsilon) & =-\frac{1728 \epsilon^{4} r^{4}-1536 \sqrt{3} \epsilon^{3} r^{4}-31104 \epsilon^{4} r^{2}+1152 \epsilon^{2} r^{4}-5184 \epsilon^{4}+2592 \epsilon^{2} r^{2}-37 r^{4}-3456 \epsilon^{2}}{24(6 \epsilon+\sqrt{3})^{2}(6 \epsilon-\sqrt{3})^{2} r^{2}}, \\
\mu_{1,5}(r, \epsilon) & =\frac{9\left(1152 \epsilon^{4} r^{2}+192 \epsilon^{4}-192 \epsilon^{2} r^{2}-r^{4}+128 \epsilon^{2}+32 r^{2}-64 r+36\right)}{8(6 \epsilon+\sqrt{3})^{2}(6 \epsilon-\sqrt{3})^{2} r^{2}} \\
\mu_{1,6}(r, \epsilon) & =-\frac{9(r+6)(r-2)^{3}}{8(6 \epsilon+\sqrt{3})^{2}(6 \epsilon-\sqrt{3})^{2} r^{2}},
\end{aligned}
$$

with the corresponding intervals $\mathcal{I}_{1}=\left[1, \frac{1+2 \sqrt{3} \epsilon}{1-\sqrt{3} \epsilon}\right), \mathcal{I}_{2}=\left[\frac{1+2 \sqrt{3} \epsilon}{1-\sqrt{3} \epsilon}, \frac{4(1-\sqrt{3} \epsilon}{3}\right), \mathcal{I}_{3}=\left[\frac{4(1-\sqrt{3} \epsilon}{3}, \frac{4(1+2 \sqrt{3} \epsilon}{3}\right)$, $\mathcal{I}_{4}=\left[\frac{4(1+2 \sqrt{3} \epsilon}{3}, \frac{3}{2(1-\sqrt{3} \epsilon)}\right), \mathcal{I}_{5}=\left[\frac{3}{2(1-\sqrt{3} \epsilon)}, 2\right)$ and $\mathcal{I}_{6}=[2, \infty)$.

For $\epsilon \in[(7 \sqrt{3}-3 \sqrt{15}) / 12, \sqrt{3} / 12), \mu_{A}(r, \epsilon)=\sum_{j=1}^{6} \mu_{2, j}(r, \epsilon) \mathbf{I}\left(r \in \mathcal{I}_{j}\right)$ where $\mu_{2, j}(r, \epsilon)=\mu_{1, j}(r, \epsilon)$ for $j=1,3,4,5,6$ and

$$
\begin{aligned}
\mu_{2,2}(r, \epsilon) & =\left[-3456 \epsilon^{2} r^{4}+111 r^{4}-5184 \epsilon^{4} r^{4}+4608 \sqrt{3} \epsilon^{3} r^{4}-336 \sqrt{3} \epsilon r^{3}-168 r^{3}-13824 \epsilon^{4} r^{3}\right. \\
& +4608 \sqrt{3} \epsilon^{3} r^{3}+3456 \epsilon^{2} r^{3}+144 r^{2}-6912 \sqrt{3} \epsilon^{3} r^{2}-3888 \epsilon^{2} r^{2}+576 \sqrt{3} \epsilon r^{2} \\
& \left.+25920 \epsilon^{4} r^{2}+3168 \epsilon^{4}+2880 \epsilon^{2}-256 \sqrt{3} \epsilon-32-3072 \sqrt{3} \epsilon^{3}\right] /\left[36(\sqrt{3}+6 \epsilon)^{2}(-6 \epsilon+\sqrt{3})^{2} r^{2}\right]
\end{aligned}
$$

with the corresponding intervals $\mathcal{I}_{1}=\left[1, \frac{4(1-\sqrt{3} \epsilon)}{3}\right), \mathcal{I}_{2}=\left[\frac{4(1-\sqrt{3} \epsilon)}{3}, \frac{1+2 \sqrt{3} \epsilon}{1-\sqrt{3} \epsilon}\right), \mathcal{I}_{3}=\left[\frac{1+2 \sqrt{3} \epsilon}{1-\sqrt{3} \epsilon}, \frac{4(1+2 \sqrt{3} \epsilon}{3}\right)$, $\mathcal{I}_{4}=\left[\frac{4(1+2 \sqrt{3} \epsilon}{3}, \frac{3}{2(1-\sqrt{3} \epsilon)}\right), \mathcal{I}_{5}=\left[\frac{3}{2(1-\sqrt{3} \epsilon)}, 2\right)$ and $\mathcal{I}_{6}=[2, \infty)$.

For $\epsilon \in[\sqrt{3} / 12, \sqrt{3} / 3), \mu_{A}(r, \epsilon)=\sum_{j=1}^{3} \mu_{3, j}(r, \epsilon) \mathbf{I}\left(r \in \mathcal{I}_{j}\right)$ where

$$
\begin{aligned}
\mu_{3,1}(r, \epsilon) & =\frac{2 r^{2}-1}{6 r^{2}}, \\
\mu_{3,2}(r, \epsilon) & =\left[432 \epsilon^{4} r^{4}+1152 \epsilon^{4} r^{3}-576 \sqrt{3} \epsilon^{3} r^{4}+1296 \epsilon^{4} r^{2}-960 \sqrt{3} \epsilon^{3} r^{3}+864 \epsilon^{2} r^{4}-864 \sqrt{3} \epsilon^{3} r^{2}\right. \\
& +576 \epsilon^{2} r^{3}-192 \sqrt{3} \epsilon r^{4}-360 \epsilon^{4}+648 \epsilon^{2} r^{2}+64 \sqrt{3} \epsilon r^{3}+48 r^{4}+192 \sqrt{3} \epsilon^{3}-144 \sqrt{3} \epsilon r^{2} \\
& \left.-64 r^{3}-504 \epsilon^{2}+72 r^{2}+88 \sqrt{3} \epsilon-25\right] /\left[16(3 \epsilon-\sqrt{3})^{4} r^{2}\right], \\
\mu_{3,3}(r, \epsilon) & =-\frac{-54 \epsilon^{2} r^{2}+36 \sqrt{3} \epsilon r^{2}+15 \epsilon^{2}-18 r^{2}+2 \sqrt{3} \epsilon+20}{6(-3 \epsilon+\sqrt{3})^{2} r^{2}},
\end{aligned}
$$

with the corresponding intervals $\mathcal{I}_{1}=\left[1, \frac{1+2 \sqrt{3} \epsilon}{2(1-\sqrt{3} \epsilon)}\right), \mathcal{I}_{3}=\left[\frac{1+2 \sqrt{3} \epsilon}{2(1-\sqrt{3} \epsilon)}, \frac{3}{2(1-\sqrt{3} \epsilon)}\right), \mathcal{I}_{5}=\left[\frac{3}{2(1-\sqrt{3} \epsilon)}, \infty\right)$. 


\section{Appendix 3: $\mu(r, \epsilon)$ and $\nu(r, \epsilon)$ for Segregation and Association Al- ternatives with Sample $\epsilon$ values}

With $\epsilon=\sqrt{3} / 4, r \in[1,2), \mu_{S}(r, \sqrt{3} / 4)=\left\{\begin{array}{ll}-\frac{67}{54} r^{2}+\frac{40}{9} r-3 & \text { for } \quad r \in[1,3 / 2) \\ \frac{7 r^{4}-48 r^{3}+122 r^{2}-128 r+48}{2 r^{2}} & \text { for } \quad r \in[3 / 2,2)\end{array}\right.$ and $\nu_{S}(r, \sqrt{3} / 4)=\sum_{j=1}^{5} \nu_{j}(r, \sqrt{3} / 4) \mathbf{I}\left(\mathcal{I}_{j}\right)$ where

$$
\begin{aligned}
\nu_{1}(r, \sqrt{3} / 4) & =-\left[14285 r^{7}-28224 r^{6}-233266 r^{5}+1106688 r^{4}-2021199 r^{3}+1876608 r^{2}\right. \\
& -880794 r+165888] /[3645 r], \\
\nu_{2}(r, \sqrt{3} / 4) & =-\left[14285 r^{10}-28224 r^{9}-233266 r^{8}+1106688 r^{7}-1234767 r^{6}-3431808 r^{5}\right. \\
& \left.+14049126 r^{4}-22228992 r^{3}+18895680 r^{2}-8503056 r+1594323\right] /\left[3645 r^{4}\right], \\
\nu_{3}(r, \sqrt{3} / 4) & =-\left[14285 r^{10}-28224 r^{9}-233266 r^{8}+1106688 r^{7}-2545713 r^{6}+5903280 r^{5}\right. \\
& \left.-13456044 r^{4}+20636208 r^{3}-18305190 r^{2}+8503056 r-1594323\right] /\left[3645 r^{4}\right], \\
\nu_{4}(r, \sqrt{3} / 4) & =\left[104920 r^{8}-111072 r^{7}+1992132 r^{6}-15844032 r^{5}+50174640 r^{4}+6377292\right. \\
& \left.-34012224 r+73220760 r^{2}-81881280 r^{3}+1909 r^{10}-27072 r^{9}\right] /\left[14580 r^{4}\right], \\
\nu_{5}(r, \sqrt{3} / 4) & =-\left[-1187904 r^{5}+1331492 r^{6}+433304 r^{2}+611163 r^{10}-850240 r^{9}-198144 r\right. \\
& +955392 r^{4}-705536 r^{3}-387680 r^{11}+1118472 r^{8}-1308960 r^{7}+175984 r^{12} \\
& \left.-46176 r^{13}+5120 r^{14}+56016\right] /\left[20 r^{4}\right],
\end{aligned}
$$

and the corresponding intervals are $\mathcal{I}_{1}=\left[1, \frac{9}{8}\right), \mathcal{I}_{2}=[9 / 8,9 / 7), \mathcal{I}_{3}=[9 / 7,4 / 3), \mathcal{I}_{4}=[4 / 3,3 / 2), \mathcal{I}_{5}=$ $[3 / 2,2)$. where

With $\epsilon=\sqrt{3} / 12, \mu_{A}(r, \sqrt{3} / 12)=\left\{\begin{array}{lll}\frac{6 r^{4}-16 r^{3}+18 r^{2}-5}{18 r^{2}} & \text { for } & r \in[1,2) \\ -\frac{37}{18} r^{-2}+1 & \text { for } & r \in[2, \infty)\end{array}\right.$ and $\nu_{A}(r, \sqrt{3} / 12)=\sum_{j=1}^{3} \nu_{j}(r, \sqrt{3} / 12) \mathbf{I}\left(\mathcal{I}_{j}\right)$

$$
\begin{aligned}
\nu_{1}(r, \sqrt{3} / 12) & =\left[10 r^{12}-96 r^{11}+240 r^{10}+192 r^{9}-1830 r^{8}+3360 r^{7}-2650 r^{6}+240 r^{5}+1383 r^{4}\right. \\
& \left.-1280 r^{3}+540 r^{2}-144 r+35\right] /\left[405 r^{6}\right], \\
\nu_{2}(r, \sqrt{3} / 12) & =\left[10 r^{12}-96 r^{11}+240 r^{10}+192 r^{9}-1670 r^{8}+2784 r^{7}-2650 r^{6}+2400 r^{5}-1047 r^{4}\right. \\
& \left.-1280 r^{3}+1269 r^{2}-144 r+35\right] /\left[405 r^{6}\right], \\
\nu_{3}(r, \sqrt{3} / 12) & =\frac{537 r^{4}-683 r^{2}-2448 r+1315}{405 r^{6}} .
\end{aligned}
$$

The corresponding intervals are $\mathcal{I}_{1}=[1,3 / 2), \mathcal{I}_{2}=[3 / 2,2), \mathcal{I}_{3}=[2, \infty)$.

\section{Appendix 4: Proof of Corollary 1}

In the multiple triangle case,

$$
\mu(r, J)=\mathbf{E}\left[\rho_{n}(r)\right]=\frac{1}{n(n-1)} \sum_{i<j} \sum_{\mathbf{E}} \mathbf{E}\left[h_{i j}\right]=\frac{1}{2} \mathbf{E}\left[h_{12}\right]=\mathbf{E}\left[I\left(A_{12}\right)\right]=P\left(A_{12}\right)=P\left(X_{2} \in N_{\mathcal{Y}}^{r}\left(X_{1}\right)\right) .
$$


But, by definition of $N_{\mathcal{Y}}^{r}(\cdot), P\left(X_{2} \in N_{\mathcal{Y}}^{r}\left(X_{1}\right)\right)=0$ if $X_{1}$ and $X_{2}$ are in different triangles. So by the law of total probability

$$
\begin{aligned}
\mu(r, J) & :=P\left(X_{2} \in N_{\mathcal{Y}}^{r}\left(X_{1}\right)\right)=\sum_{j=1}^{J} P\left(X_{2} \in N_{\mathcal{Y}}^{r}\left(X_{1}\right) \mid\left\{X_{1}, X_{2}\right\} \subset T_{j}\right) P\left(\left\{X_{1}, X_{2}\right\} \subset T_{j}\right) \\
& =\sum_{j=1}^{J} \mu(r) P\left(\left\{X_{1}, X_{2}\right\} \subset T_{j}\right) \quad\left(\text { since } P\left(X_{2} \in N_{\mathcal{Y}}^{r}\left(X_{1}\right) \mid\left\{X_{1}, X_{2}\right\} \subset T_{j}\right)=\mu(r)\right) \\
& =\mu(r) \sum_{j=1}^{J}\left(A\left(T_{j}\right) / A\left(C_{H}(\mathcal{Y})\right)\right)^{2} \quad\left(\text { since } P\left(\left\{X_{1}, X_{2}\right\} \subset T_{j}\right)=\left(A\left(T_{j}\right) / A\left(C_{H}(\mathcal{Y})\right)\right)^{2}\right)
\end{aligned}
$$

Letting $w_{j}:=A\left(T_{j}\right) / A\left(C_{H}(\mathcal{Y})\right)$, we get $\mu(r, J)=\mu(r) \cdot\left(\sum_{j=1}^{J} w_{j}^{2}\right)$ where $\mu(r)$ is given by equation (8).

Furthermore, the asymptotic variance is

$$
\begin{aligned}
\nu(r, J)= & \mathbf{E}\left[h_{12} h_{13}\right]-\mathbf{E}\left[h_{12}\right] \mathbf{E}\left[h_{13}\right] \\
= & P\left(\left\{X_{2}, X_{3}\right\} \subset N_{\mathcal{Y}}^{r}\left(X_{1}\right)\right)+2 P\left(X_{2} \in N_{\mathcal{Y}}^{r}\left(X_{1}\right), X_{3} \in \Gamma_{1}\left(X_{1}, N_{\mathcal{Y}}^{r}\right)\right) \\
& +P\left(\left\{X_{2}, X_{3}\right\} \subset \Gamma_{1}\left(X_{1}, N_{\mathcal{Y}}^{r}\right)\right)-4(\mu(r, J))^{2} .
\end{aligned}
$$

Then for $J>1$, we have

$$
\begin{aligned}
P\left(\left\{X_{2}, X_{3}\right\} \subset N_{\mathcal{Y}}^{r}\left(X_{1}\right)\right) & =\sum_{j=1}^{J} P\left(\left\{X_{2}, X_{3}\right\} \subset N_{\mathcal{Y}}^{r}\left(X_{1}\right) \mid\left\{X_{1}, X_{2}, X_{3}\right\} \subset T_{j}\right) P\left(\left\{X_{1}, X_{2}, X_{3}\right\} \subset T_{j}\right) \\
& =\sum_{j=1}^{J} P_{2 N}^{r}\left(A\left(T_{j}\right) / A\left(C_{H}(\mathcal{Y})\right)\right)^{3}=P_{2 N}^{r}\left(\sum_{j=1}^{J} w_{j}^{3}\right) .
\end{aligned}
$$

Similarly, $P\left(X_{2} \in N_{\mathcal{Y}}^{r}\left(X_{1}\right), X_{3} \in \Gamma_{1}\left(X_{1}, N_{\mathcal{Y}}^{r}\right)\right)=P_{M}^{r}\left(\sum_{j=1}^{J} w_{j}^{3}\right)$ and $P\left(\left\{X_{2}, X_{3}\right\} \subset \Gamma_{1}\left(X_{1}, N_{\mathcal{Y}}^{r}\right)\right)=P_{2 G}^{r}\left(\sum_{j=1}^{J} w_{j}^{3}\right)$, hence, $\nu(r, J)=\left(P_{2 N}^{r}+2 P_{M}^{r}+P_{2 G}^{r}\right)\left(\sum_{j=1}^{J} w_{j}^{3}\right)-4(\mu(r, J))^{2}=\nu(r)\left(\sum_{j=1}^{J} w_{j}^{3}\right)+4 \mu(r)^{2}\left(\sum_{j=1}^{J} w_{j}^{3}-\right.$ $\left.\left(\sum_{j=1}^{J} w_{j}^{2}\right)^{2}\right)$, so conditional on $\mathcal{W}$, if $\nu(r, J)>0$ then $\sqrt{n}\left(\rho_{n}(r)-\mu(r, J)\right) \stackrel{\mathcal{L}}{\longrightarrow} \mathcal{N}(0, \nu(r, J))$. 